\title{
A degree sequence version of the Kühn-Osthus tiling theorem
}

\author{
Joseph Hyde Andrew Treglown \\ School of Mathematics \\ University of Birmingham \\ Birmingham, U.K. \\ \{JFH337, A.C.Treglown\}@bham.ac.uk
}

Submitted: Sep 3, 2019; Accepted: Jul 13, 2020; Published: Sep 4, 2020

(C) The authors. Released under the CC BY license (International 4.0).

\begin{abstract}
A fundamental result of Kühn and Osthus [The minimum degree threshold for perfect graph packings, Combinatorica, 2009] determines up to an additive constant the minimum degree threshold that forces a graph to contain a perfect $H$-tiling. We prove a degree sequence version of this result which allows for a significant number of vertices to have lower degree.
\end{abstract}

Mathematics Subject Classifications: 05C35, 05C70

\section{Introduction}

\subsection{Minimum degree conditions forcing tilings}

A substantial branch of extremal graph theory concerns the study of tilings. Given two graphs $H$ and $G$, an $H$-tiling in $G$ is a collection of vertex-disjoint copies of $H$ in $G$. An $H$-tiling is called perfect if it covers all the vertices of $G$. Perfect $H$-tilings are also often referred to as $H$-factors, perfect $H$-packings or perfect $H$-matchings.

In the case when $H$ has a component on at least 3 vertices, the decision problem of whether a graph contains a perfect $H$-tiling is NP-complete [6]. Thus, there has been a focus on establishing sufficient conditions to force a perfect $H$-tiling. The seminal HajnalSzemerédi theorem [5] characterises the minimum degree that ensures a graph contains a perfect $K_{r}$-tiling.

Theorem 1 (Hajnal and Szemerédi [5]). Every graph $G$ whose order $n$ is divisible by $r$ and whose minimum degree satisfies $\delta(G) \geqslant(1-1 / r) n$ contains a perfect $K_{r}$-tiling. Moreover, there are $n$-vertex graphs $G$ with $\delta(G)=(1-1 / r) n-1$ that do not contain a perfect $K_{r}$-tiling. 
The following result of Alon and Yuster [1] shows that any sufficiently large graph $G$ with minimum degree slightly above that in Theorem 1 in fact contains a perfect $H$-tiling for any graph $H$ with $\chi(H)=r$.

Theorem 2 (Alon and Yuster [1]). Suppose that $\gamma>0$ and $H$ is a graph with $\chi(H)=r$. Then there exists an integer $n_{0}=n_{0}(\gamma, H)$ such that the following holds. If $G$ is a graph whose order $n \geqslant n_{0}$ is divisible by $|H|$ and

$$
\delta(G) \geqslant(1-1 / r+\gamma) n
$$

then $G$ contains a perfect $H$-tiling.

For many graphs $H$ the minimum degree condition in Theorem 2 is best-possible up to the term $\gamma n$. Indeed, for many graphs $H$ there are so-called divisibility barrier constructions $G$ on $n$ vertices that have minimum degree $(1-1 / \chi(H)) n-1$ but fail to contain a perfect $H$-tiling (see [15, Section 2]). However, Komlós, Sárközy and Szemerédi [12] proved that the term $\gamma n$ in Theorem 2 can be replaced with a constant dependent only on $H$. Further, as discussed shortly, Kühn and Osthus $[14,15]$ proved that there are also many graphs $H$ for which one can significantly reduce the minimum degree condition in Theorem 2.

In a related direction, Komlós [10] showed that if one only requires an $H$-tiling covering almost all vertices in the host graph, then one can replace the $\chi(H)$-term in the minimum degree condition of the Alon-Yuster theorem by the so-called critical chromatic number $\chi_{c r}(H)$ of $H$. Here $\chi_{c r}(H)$ is defined as

$$
\chi_{c r}(H):=(\chi(H)-1) \frac{|H|}{|H|-\sigma(H)},
$$

where $\sigma(H)$ denotes the size of the smallest possible colour class in any $\chi(H)$-colouring of $H$. Note that all graphs $H$ satisfy $\chi(H)-1<\chi_{c r}(H) \leqslant \chi(H)$ and $\chi_{c r}(H)=\chi(H)$ precisely when every $\chi(H)$-colouring $c$ of $H$ is balanced (i.e. the colour classes of $c$ have the same size).

Theorem 3 (Komlós [10]). Let $\eta>0$ and let $H$ be a graph. Then there exists an integer $n_{0}=n_{0}(\eta, H)$ such that every graph $G$ on $n \geqslant n_{0}$ vertices with

$$
\delta(G) \geqslant\left(1-\frac{1}{\chi_{c r}(H)}\right) n
$$

contains an $H$-tiling covering all but at most $\eta n$ vertices.

Note that the minimum degree condition in Theorem 3 is best-possible in the sense that one cannot replace the $\left(1-1 / \chi_{c r}(H)\right)$ term here with any smaller fixed constant (this is a consequence of $[10$, Theorem 7$])$. Further, for any $x \in(0,1)$ and sufficiently large $n$, Komlós [10] determined the minimum degree threshold that ensures a graph $G$ on $n$ vertices contains an $H$-tiling covering at least $x n$ vertices. Shoukoufandeh and Zhao [18] 
later proved that the number of uncovered vertices in Theorem 3 can be reduced to a constant dependent only on $H$.

Kühn and Osthus $[14,15]$ showed that for many graphs $H$, a minimum degree slightly above that in Komlós' theorem actually ensures a perfect $H$-tiling. To state their result we need to introduce some notation. We say that a colouring of $H$ is optimal if it uses exactly $\chi(H)=: r$ colours. Let $C_{H}$ denote the set of all optimal colourings of $H$. Given an optimal colouring $c$ of $H$, let $x_{c, 1} \leqslant x_{c, 2} \leqslant \cdots \leqslant x_{c, r}$ denote the sizes of the colour classes of $c$. We write

$$
\mathcal{D}(c):=\left\{x_{c, i+1}-x_{c, i} \mid i=1, \ldots, r-1\right\},
$$

and let

$$
\mathcal{D}(H):=\bigcup_{c \in C_{H}} \mathcal{D}(c)
$$

We denote by $\operatorname{hcf}_{\chi}(H)$ the highest common factor of all integers in $\mathcal{D}(H)$. If $\mathcal{D}(H)=\{0\}$ then we define $\operatorname{hcf}_{\chi}(H):=\infty$. We write $\operatorname{hcf}_{c}(H)$ for the highest common factor of all the orders of components of $H$. For non-bipartite graphs $H$ we say that $\operatorname{hcf}(H)=1$ if $\operatorname{hcf}_{\chi}(H)=1$. If $\chi(H)=2$ then we say $\operatorname{hcf}(H)=1$ if $\operatorname{hcf}_{c}(H)=1$ and $\operatorname{hcf}_{\chi}(H) \leqslant 2$. (See [15] for some examples.) Set

$$
\chi^{*}(H):= \begin{cases}\chi_{c r}(H) & \text { if } \operatorname{hcf}(H)=1 \\ \chi(H) & \text { otherwise. }\end{cases}
$$

Also let $\delta(H, n)$ denote the smallest integer $k$ such that every graph $G$ whose order $n$ is divisible by $|H|$ and with $\delta(G) \geqslant k$ contains a perfect $H$-tiling.

When $\operatorname{hcf}(H)=1$, Kühn and Osthus showed that $\chi_{c r}(H)$ is the parameter governing the minimum degree condition that ensures a perfect $H$-tiling. When $\operatorname{hcf}(H) \neq 1, \chi(H)$ instead is the relevant parameter.

Theorem 4 (Kühn and Osthus [15]). For every graph $H$ there exists a constant $C=C(H)$ such that

$$
\left(1-\frac{1}{\chi^{*}(H)}\right) n-1 \leqslant \delta(H, n) \leqslant\left(1-\frac{1}{\chi^{*}(H)}\right) n+C .
$$

Intuitively speaking, graphs $H$ with $\operatorname{hcf}(H)=1$ avoid certain divisibility barrier problems when seeking a perfect $H$-tiling, thus ensuring the lower threshold in this case in Theorem 4. Earlier Kühn and Osthus [14] had proven a version of Theorem 4 for graphs $H$ with $\chi(H) \geqslant 3$ and $\operatorname{hcf}(H)=1$; there though the constant $C(H)$ was replaced with a linear error term. We now state this result explicitly for future reference.

Theorem 5 (Kühn and Osthus [14]). Let $\eta>0$ and $H$ be a graph with $\operatorname{hcf}_{\chi}(H)=1$ and $\chi(H)=: r \geqslant 3$. Then there exists an integer $n_{0}=n_{0}(\eta, H)$ such that the following holds. Let $G$ be a graph on $n \geqslant n_{0}$ vertices such that $|H|$ divides $n$ and

$$
\delta(G) \geqslant\left(1-\frac{1}{\chi_{c r}(H)}+\eta\right) n .
$$

Then $G$ contains a perfect $H$-tiling. 


\subsection{Degree sequence conditions forcing tilings}

As discussed in the previous subsection, the minimum degree conditions in each of the Hajnal-Szemerédi theorem, Komlós' theorem and the Kühn-Osthus theorem are essentially best-possible. However, this does not mean one cannot seek significant strengthenings of these results. For example, Kierstead and Kostochka [9] proved an Ore-type generalisation of Theorem 1 where now one replaces the minimum degree condition with the condition that the sum of the degrees of every pair of non-adjacent vertices in $G$ is at least $2(1-1 / r) n-1$.

The focus of this paper concerns degree sequence conditions that force a perfect $\mathrm{H}$ tiling. The study of degree sequence results for tilings was initiated in [2]. In particular, a conjecture on a degree sequence strengthening of the Hajnal-Szemerédi theorem was raised [2, Conjecture 7], as well as a degree sequence version of the Alon-Yuster theorem [2, Conjecture 8]. In [20] the second author proved the latter conjecture (also yielding an asymptotic version of Conjecture 7 from [2]).

Theorem 6 (Treglown [20]). Suppose that $\eta>0$ and $H$ is a graph with $\chi(H)=: r \geqslant 2$. Then there exists an integer $n_{0}=n_{0}(\eta, H)$ such that the following holds. If $G$ is a graph whose order $n \geqslant n_{0}$ is divisible by $|H|$, and whose degree sequence $d_{1} \leqslant \cdots \leqslant d_{n}$ satisfies

$$
d_{i} \geqslant(r-2) n / r+i+\eta n \text { for all } 1 \leqslant i \leqslant n / r,
$$

then $G$ contains a perfect $H$-tiling.

Theorem 6 is a significant strengthening of the Alon-Yuster theorem as it allows for $n / r$ vertices to have degree (significantly) below that required in the latter. Further Theorem 6 provides the first piece of a degree sequence analogue of the Kühn-Osthus theorem.

The main result in this paper deals with the remaining part of this problem, providing a degree sequence condition that forces a perfect $H$-tiling for graphs with $\operatorname{hcf}(H)=1$.

Theorem 7. Let $\eta>0$ and $H$ be a graph with $\operatorname{hcf}(H)=1$ and $\chi(H)=: r \geqslant 2$. Let $\sigma:=\sigma(H), h:=|H|$ and $\omega:=(h-\sigma) /(r-1)$. Then there exists an integer $n_{1}=n_{1}(\eta, H)$ such that the following holds. Let $G$ be a graph on $n \geqslant n_{1}$ vertices such that $h$ divides $n$ and $G$ has degree sequence $d_{1} \leqslant \cdots \leqslant d_{n}$ such that

$$
d_{i} \geqslant\left(1-\frac{\omega+\sigma}{h}\right) n+\frac{\sigma}{\omega} i+\eta n \text { for all } 1 \leqslant i \leqslant \frac{\omega n}{h} .
$$

Then $G$ contains a perfect $H$-tiling.

Note that here $\omega$ is the average size of the colour classes of $H$ after excluding one of minimal size $\sigma(H)$. Observe that when $i=\omega n / h$, we have

$$
d_{\frac{\omega n}{h}} \geqslant\left(1-\frac{\omega}{h}+\eta\right) n=\left(1-\frac{1}{\chi_{c r}(H)}+\eta\right) n .
$$


Hence, Theorem 7 is a strengthening of Theorem 5. Note that Theorem 7 applies to all graphs $H$ with $\operatorname{hcf}(H)=1$, not just graphs $H$ with $\chi(H) \geqslant 3$ and $\operatorname{hcf}_{\chi}(H)=1$ (as in Theorem 5). Moreover, Theorem 7 (and Theorem 6) is best-possible for many graphs $H$ in the sense that we cannot replace the $\eta n$-term with a $o(\sqrt{n})$-term (see Proposition 12 ). Theorem 7 is also best possible for all graphs $H$ in the sense that there are $n$-vertex graphs $G$ with only slightly more than $\omega n / h$ vertices with degree (slightly) below $(1-\omega / h+\eta) n$ that do not contain a perfect $H$-tiling (see Proposition 15 ).

Thus, it is not possible to allow significantly more 'small' degree vertices in Theorem 7. Extremal examples are discussed in more detail in Section 3.2.

Combining Theorem 7 with Theorem 6 we obtain the following degree sequence version of the Kühn-Osthus theorem (Theorem 4).

Theorem 8. Let $\eta>0$ and $H$ be a graph with $\chi(H)=: r \geqslant 2$. Let $\sigma:=\sigma(H), h:=|H|$ and $\omega:=(h-\sigma) /(r-1)$. Then there exists an integer $n_{1}=n_{1}(\eta, H)$ such that if $G$ is a graph on $n \geqslant n_{1}$ vertices, $h$ divides $n$ and either (i) or (ii) below holds, then $G$ contains a perfect $H$-tiling.

(i) $\operatorname{hcf}(H)=1$ and $G$ has degree sequence $d_{1} \leqslant \cdots \leqslant d_{n}$ such that

$$
d_{i} \geqslant\left(1-\frac{\omega+\sigma}{h}\right) n+\frac{\sigma}{\omega} i+\eta n \text { for all } 1 \leqslant i \leqslant \frac{\omega n}{h} .
$$

(ii) $\operatorname{hcf}(H) \neq 1$ and $G$ has degree sequence $d_{1} \leqslant \ldots \leqslant d_{n}$ such that

$$
d_{i} \geqslant(r-2) n / r+i+\eta n \text { for all } 1 \leqslant i \leqslant n / r \text {. }
$$

One can in fact obtain the following generalisation of Theorem 7 in which we assume $\sigma(H) \leqslant \sigma<h / r$.

Theorem 9. Let $\eta>0$ and $H$ be a graph with $\operatorname{hcf}(H)=1$ and $\chi(H)=: r \geqslant 2$. Let $h:=|H|$. Set $\sigma \in \mathbb{R}$ such that $\sigma(H) \leqslant \sigma<h / r$ and $\omega:=(h-\sigma) /(r-1)$. Then there exists an integer $n_{1}=n_{1}(\eta, H)$ such that the following holds. Let $G$ be a graph on $n \geqslant n_{1}$ vertices such that $h$ divides $n$ and $G$ has degree sequence $d_{1} \leqslant \cdots \leqslant d_{n}$ such that

$$
d_{i} \geqslant\left(1-\frac{\omega+\sigma}{h}\right) n+\frac{\sigma}{\omega} i+\eta n \text { for all } 1 \leqslant i \leqslant \frac{\omega n}{h} .
$$

Then $G$ contains a perfect $H$-tiling.

Observe that for graphs $H$ with $\operatorname{hcf}(H)=1$, Theorem 9 interpolates between Theorems 6 and 7. In Section 7, we will prove Theorem 9 directly. The proof of Theorem 9 follows that of Theorem 5 in [14] closely. The main novelty of our proof is how we avoid divisibility barriers. For this we make use of an elementary number theoretic result for graphs with $\operatorname{hcf}(H)=1$ (see Theorem 30). We also make use of a recent degree sequence strengthening of Komlós' theorem proved by the authors and Liu [7]. 


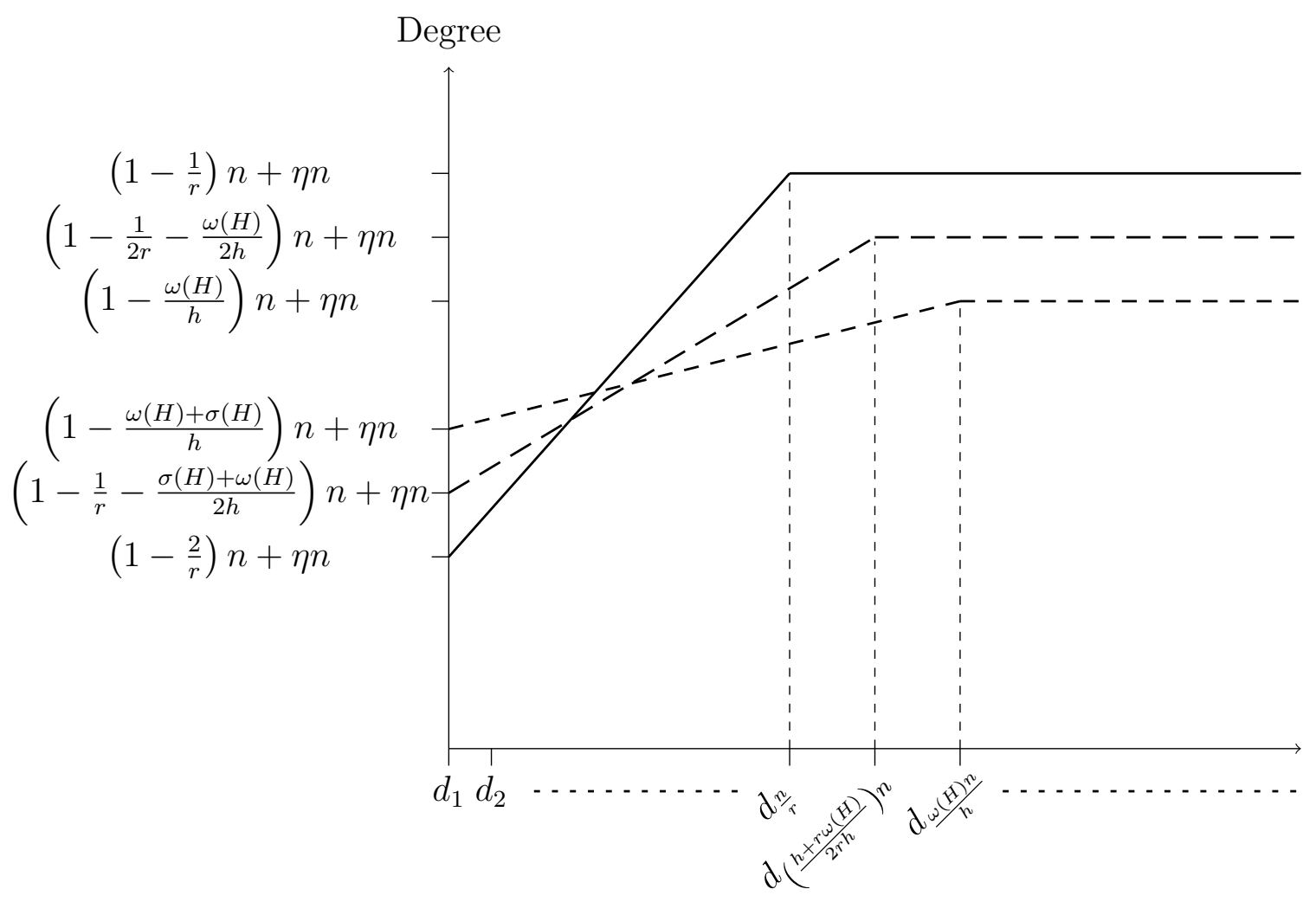

Figure 1: The degree sequence in Theorem 9 for a fixed graph $H$ given $\sigma=\sigma(H)$ (medium dashed); $\sigma=\frac{h+r \sigma(H)}{2 r}$ (long dashed); $\sigma=\frac{h}{r}$ (full).

Since the choice of $\sigma \in[\sigma(H), h / r)$ is arbitrary, note that Theorem 9 provides an infinite collection of degree sequences that force a perfect $H$-tiling. Having a higher value of $\sigma$ lowers the starting point of the degree sequence condition, but at the price of a steeper 'slope' and higher value of $d_{\omega n / h}$ (see Figure 1).

As with Theorem 7, for many graphs $H$, each of these degree sequences is best-possible in the sense that we cannot replace the $\eta n$-term with a $o(\sqrt{n})$-term (see Section 3.2). Note too that we cannot extend Theorem 9 to the case when $\sigma<\sigma(H)$. Indeed, in this case, if we set $\eta \ll 1$ then the degree sequence condition in Theorem 9 would allow all vertices in $G$ to have degree below $\left(1-1 / \chi_{c r}(H)\right) n-1$; however, we know from Theorem 4 that there are graphs $G$ that satisfy this condition and that do not contain perfect $H$-tilings.

The paper is organised as follows. In the next section we introduce some notation and definitions. In Section 3 we discuss various senses of optimality for degree sequence conditions before giving several extremal examples for Theorems 7 and 9 . We also ask whether one can improve Theorem 7 by suitably 'capping' the bounds on the degrees of the vertices (see Question 16). In Section 4 we give a number of auxiliary results and definitions relating to the regularity lemma and tilings. We then prove an elementary number 
theoretic result (Theorem 30) in Section 5 which will be a crucial tool in overcoming divisibility barriers during the proof of Theorem 9. In Section 6 we give an overview of the proof of Theorem 9 before proving it in Section 7 .

\section{Notation and Definitions}

Let $G$ be a graph. We define $V(G)$ to be the vertex set of $G$ and $E(G)$ to be the edge set of $G$. Let $X \subseteq V(G)$. Then $G[X]$ is the graph induced by $X$ on $G$ and has vertex set $X$ and edge set $E(G[X]):=\{x y \in E(G): x, y \in X\}$. We also define $G \backslash X$ to be the graph with vertex set $V(G) \backslash X$ and edge set $E(G \backslash X):=\{x y \in E(G): x, y \in V(G) \backslash X\}$. For each $x \in V(G)$, we define the neighbourhood of $x$ in $G$ to be $N_{G}(x):=\{y \in V(G): x y \in E(G)\}$ and define $d_{G}(x):=\left|N_{G}(x)\right|$. We drop the subscript $G$ if it is clear from context which graph we are considering. We write $d_{G}(x, X)$ for the number of edges in $G$ that $x$ sends to vertices in $X$. Given a subgraph $G^{\prime} \subseteq G$, we will write $d_{G}\left(x, G^{\prime}\right):=d_{G}\left(x, V\left(G^{\prime}\right)\right)$. Let $A, B \subseteq V(G)$ be disjoint. Then we define $e_{G}(A, B):=|\{x y \in E(G): x \in A, y \in B\}|$.

Let $t \in \mathbb{N}$. We define the blow-up $G(t)$ to be the graph constructed by first replacing each vertex $x \in V(G)$ by a set $V_{x}$ of $t$ vertices and then replacing each edge $x y \in E(G)$ with the edges of the complete bipartite graph with vertex sets $V_{x}$ and $V_{y}$.

We write $0<a \ll b \ll c<1$ to mean that we can choose the constants $a, b, c$ from right to left. More precisely, there exist non-decreasing functions $f:(0,1] \rightarrow(0,1]$ and $g:(0,1] \rightarrow(0,1]$ such that for all $a \leqslant f(b)$ and $b \leqslant g(c)$ our calculations and arguments in our proofs are correct. Larger hierarchies are defined similarly. Note that $a \ll b$ implies that we may assume e.g. $a<b$ or $a<b^{2}$.

\section{A discussion on the optimality of degree sequence conditions}

In this section we describe various notions concerning when a degree sequence condition is 'best-possible' in some sense. In particular, we will explain in what way our results (Theorems 7 and 9) are essentially best-possible, as well as how we may be able to strengthen these theorems further. Some of our discussion draws on the survey [3].

First we introduce a few definitions. An integer sequence $\pi=\left(d_{1} \leqslant \ldots \leqslant d_{n}\right)$ is called graphic if there exists a (simple) graph $G$ that has $\pi$ as its degree sequence. Given a graph property $P$, we say that a graphic sequence $\pi$ forces $P$ if every graph with degree sequence $\pi$ satisfies property $P$. Given a property $P$ (such as containing a Hamilton cycle or perfect $H$-tiling), the 'holy-grail' in the study of degree sequences is to establish all those graphic sequences $\pi$ that force $P$.

The following theorem of Chvátal [4] provides an extremely general condition on degree sequences that force a Hamilton cycle.

Theorem 10 (Chvátal [4]). Suppose that the degree sequence of a graph $G$ is $d_{1} \leqslant \cdots \leqslant$ $d_{n}$. If $n \geqslant 3$ and $d_{i} \geqslant i+1$ or $d_{n-i} \geqslant n-i$ for all $i<n / 2$ then $G$ is Hamiltonian.

Note that Chvátal's theorem is best-possible in the following sense: if $d_{1} \leqslant \cdots \leqslant d_{n}$ is a degree sequence that does not satisfy the condition in Theorem 10 then there exists 
a non-Hamiltonian graph $G$ whose degree sequence $d_{1}^{\prime} \leqslant \cdots \leqslant d_{n}^{\prime}$ is such that $d_{i}^{\prime} \geqslant d_{i}$ for all $1 \leqslant i \leqslant n$. (We will informally refer to a degree sequence result being best-possible in this way as a Chvátal-type result.)

Crucially note though that Chvátal's theorem does not describe all those graphic sequences that force a Hamilton cycle. For example, graphs with degree sequence $(2,2,2,2,2)$ must be Hamiltonian (in fact, are themselves simply a 5 -cycle), but do not satisfy Chvátal's condition. More generally, all $2 k$-regular graphs on $4 k+1$ vertices are Hamiltonian [17] yet their degree sequences fail the condition in Theorem 10.

\subsection{Degree sequence conditions forcing perfect $\boldsymbol{H}$-tilings}

At present, for a given fixed graph $H$, it seems out of reach to characterise those graphic degree sequences that force a perfect $H$-tiling, or obtain a Chvátal-type result in this setting. Thus, it is natural to seek degree sequence conditions that force a perfect $H$ tiling, and are best-possible in some weaker sense. For example, consider the following conjecture:

Conjecture 11 (Balogh, Kostochka and Treglown [2]). Let $n, r \in \mathbb{N}$ such that $r$ divides $n$. Suppose that $G$ is a graph on $n$ vertices with degree sequence $d_{1} \leqslant \cdots \leqslant d_{n}$ such that:

$(\alpha) d_{i} \geqslant(r-2) n / r+i$ for all $i<n / r$

(в) $d_{n / r+1} \geqslant(r-1) n / r$.

Then $G$ contains a perfect $K_{r}$-tiling.

Conjecture 11 is best-possible in the sense that there are examples (see [2, Section 4]) showing that one cannot replace $(\alpha)$ with $d_{i} \geqslant(r-2) n / r+i-1$ for even a single $i$ or $(\beta)$ with $d_{n / r+1} \geqslant(r-1) n / r-1$ and $d_{n / r+2} \geqslant(r-1) n / r$. That is, there is no room to lower the degree sequence condition further, not even by lowering a single entry by just one. (We will informally refer to a degree sequence result being best-possible in this way as a Pósa-type result.) However, Conjecture 11, if true, is likely still significantly weaker than a Chvátal-type result. For example, it is easy to see that any graph $G$ with degree sequence $d_{1} \leqslant \cdots \leqslant d_{n}$ satisfying (i) $d_{1} \geqslant r-1$; (ii) $d_{2} \geqslant n-2$; (iii) $d_{n-r+2} \geqslant n-1$ contains a perfect $K_{r}$-tiling even though the condition in Conjecture 11 is violated.

One could also ask for a Pósa-type strengthening of the Kühn-Osthus theorem (Theorem 4) for all graphs $H$. However, obtaining such a result again seems extremely difficult, not only because (the special case) Conjecture 11 is still open, but also in general because the 'correct' value of the constant $C(H)$ in Theorem 4 is not known.

\subsection{Extremal examples for Theorems 7 and 9}

Despite the aforementioned challenges, in this paper we have provided degree sequence conditions that force a perfect $H$-tiling, and are best-possible in various ways. The following 3 extremal examples demonstrate this. The first shows that we cannot significantly lower every term in the degree sequence conditions of Theorems 7 and 9 and still ensure a 
perfect $H$-tiling for complete $r$-partite graphs $H$. The second shows that that the 'slope' of the degree sequence in Theorem 7 is best possible for so-called bottle graphs. The third demonstrates that for any graph $H$, to ensure a perfect $H$-tiling (or even an 'almost' perfect $H$-tiling) in a graph $G$ on $n$ vertices we cannot have significantly more than $\omega n / h$ vertices that have degree below $\left(1-\frac{1}{\chi_{c r}(H)}+\eta\right) n$.

Extremal Example 1. The following construction (a simple adaption of [20, Proposition 3.1]) demonstrates that for most complete $r$-partite graphs $H$, one cannot replace the $\eta n$ term in Theorems 7 and 9 with a $o(\sqrt{n})$-term.

Proposition 12. Let $r \geqslant 3$ and $H:=K_{t_{1}, \ldots, t_{r}}$ with $t_{i} \geqslant 2$ (for all $1 \leqslant i \leqslant r$ ). Let $h:=|H|$. Set $\sigma \in \mathbb{R}$ such that $\sigma(H) \leqslant \sigma<h / r$ and $\omega:=(h-\sigma) /(r-1)$. Let $n \in \mathbb{N}$ be sufficiently large so that $\sqrt{n}$ is an integer that is divisible by $6 h^{2}$. Set $C:=\sqrt{n} / 3 h^{2}$. Then there exists a graph $G$ on $n$ vertices whose degree sequence $d_{1} \leqslant \cdots \leqslant d_{n}$ satisfies

$$
d_{i} \geqslant\left(1-\frac{\omega+\sigma}{h}\right) n+\frac{\sigma}{\omega} i+C \text { for all } 1 \leqslant i \leqslant \frac{\omega n}{h}
$$

but such that $G$ does not contain a perfect $H$-tiling.

Proof. Let $G$ denote the graph on $n$ vertices consisting of $r$ vertex classes $V_{1}, \ldots, V_{r}$ with $\left|V_{1}\right|=1,\left|V_{2}\right|=\omega n / h+1+C r,\left|V_{3}\right|=(\sigma+\omega) n / h-2-3 C$ and $\left|V_{i}\right|=\omega n / h-C$ if $4 \leqslant i \leqslant r$ and which contains the following edges:

- All possible edges with an endpoint in $V_{3}$ and the other endpoint in $V(G) \backslash V_{1}$. (In particular, $G\left[V_{3}\right]$ is complete.);

- All edges with an endpoint in $V_{2}$ and the other endpoint in $V(G) \backslash V_{2}$;

- All edges with an endpoint in $V_{i}$ and the other endpoint in $V(G) \backslash V_{i}$ for $4 \leqslant i \leqslant r$;

- There are $\sqrt{n} / 2$ vertex-disjoint stars in $V_{2}$, each of size $\left\lfloor 2\left|V_{2}\right| / \sqrt{n}\right\rfloor$ or $\left\lceil 2\left|V_{2}\right| / \sqrt{n}\right\rceil$, which cover all of $V_{2}$.

In particular, note that the vertex $v \in V_{1}$ sends all possible edges to $V(G) \backslash V_{3}$ but no edges to $V_{3}$.

Let $d_{1} \leqslant \ldots \leqslant d_{n}$ denote the degree sequence of $G$. Notice that every vertex in $V_{i}$ for $3 \leqslant i \leqslant r$ has degree at least $(1-\omega / h) n+C$. Note that $\left\lfloor 2\left|V_{2}\right| / \sqrt{n}\right\rfloor \geqslant 2 \sqrt{n} / h=6 C h \geqslant$ $6 C r$. Thus, there are $\sqrt{n} / 2$ vertices in $V_{2}$ of degree at least

$$
(1-\omega / h) n-1-C r+(6 C r-1) \geqslant(1-\omega / h) n+C .
$$

The remaining $\omega n / h+1+C r-\sqrt{n} / 2 \leqslant \omega n / h-\sqrt{n} / 3-1$ vertices in $V_{2}$ have degree at least

$$
(1-\omega / h) n-C r \geqslant(1-\omega / h) n-\sigma \sqrt{n} / 3 \omega+C .
$$

Since $d_{G}(v) \geqslant\left(1-\frac{\omega+\sigma}{h}\right) n+C+\sigma / \omega$ for the vertex $v \in V_{1}$ we have that

$$
d_{i} \geqslant\left(1-\frac{\omega+\sigma}{h}\right) n+\frac{\sigma}{\omega} i+C \text { for all } 1 \leqslant i \leqslant \frac{\omega n}{h} .
$$


Suppose that $v \in V_{1}$ lies in a copy $H^{\prime}$ of $H$ in $G$. Then by construction of $G$, two of the vertex classes $U_{1}, U_{2}$ of $H^{\prime}$ must lie entirely in $V_{2}$. By definition of $H, H^{\prime}\left[U_{1} \cup U_{2}\right]$ contains a path of length 3. However, $G\left[V_{2}\right]$ does not contain a path of length 3 , a contradiction. Thus, $v$ does not lie in a copy of $H$ and so $G$ does not contain a perfect $H$-tiling.

Extremal Example 2. We require the following definitions. Let $t \in \mathbb{N}$. We will refer to a vertex class of size $t$ of $G$ as a $t$-class of $G$. Set $r, \sigma, \omega \in \mathbb{N}$ and $\sigma<\omega$. We define the $r$-partite bottle graph $B$ with neck $\sigma$ and width $\omega$ to be the complete $r$-partite graph with one $\sigma$-class and $(r-1) \omega$-classes.

Let $\eta>0$ be fixed. Let $B$ be an $r$-partite bottle graph with neck $\sigma$ and width $\omega$. The following extremal example (adapted from Proposition 3.1 in [7]) $G$ on $n$ vertices demonstrates that Theorem 7 is best possible for such graphs $B$, in the sense that $G$ satisfies the degree sequence of Theorem 7 except for a small linear part that only just fails the degree sequence, but does not contain a perfect $B$-tiling. In fact, $G$ does not contain a $B$-tiling that covers all but at most $\eta n$ vertices.

Proposition 13. Let $\eta>0$ be fixed and $n \in \mathbb{N}$ such that $0<1 / n \ll \eta \ll 1$. Let $r \geqslant 3$ be an integer. Let $B$ be an $r$-partite bottle graph with neck $\sigma$ and width $\omega$, where $b:=|B|$. Additionally assume that $b$ divides $n$. Then for any $1 \leqslant k<\omega n / b-(r b+1) \eta n$, there exists a graph $G$ on $n$ vertices whose degree sequence $d_{1} \leqslant \cdots \leqslant d_{n}$ satisfies

$$
\begin{gathered}
d_{i} \geqslant\left(1-\frac{\omega+\sigma}{b}\right) n+\frac{\sigma}{\omega} i+\eta n \text { for all } i \in\{1, \ldots, k-1, k+r b \eta n+1, \ldots, \omega n / b\}, \\
d_{i}=\left(1-\frac{\omega+\sigma}{b}\right) n+\left\lceil\frac{\sigma}{\omega} k\right\rceil+\eta n \text { for all } k \leqslant i \leqslant k+r b \eta n,
\end{gathered}
$$

but such that $G$ does not contain a B-tiling covering all but at most $\eta n$ vertices.

Proof. Let $G$ be the graph on $n$ vertices with $r+1$ vertex classes $V_{1}, \ldots, V_{r+1}$ where

- $\left|V_{1}\right|=\sigma n / b$;

- $\left|V_{2}\right|=\omega n / b-\eta n ;$

- $\left|V_{3}\right|=\ldots=\left|V_{r}\right|=\omega n / b-(\eta n+1) ;$

- $\left|V_{r+1}\right|=(r-1)(\eta n+1)-1$.

Label the vertices of $V_{1}$ as $a_{1}, a_{2}, \ldots, a_{\sigma n / b}$. Similarly, label the vertices of $V_{2}$ as $c_{1}, c_{2}, \ldots, c_{\omega n / b-\eta n}$. The edge set of $G$ is constructed through the following process.

Initially, let $G$ have the following edges:

- All edges with an endpoint in $V_{1}$ and the other endpoint in $V(G) \backslash V_{2}$, in particular $G\left[V_{1}\right]$ is complete;

- All edges with an endpoint in $V_{i}$ and the other endpoint in $V(G) \backslash\left(V_{1} \cup V_{i}\right)$ for $2 \leqslant i \leqslant r+1$ 
- All edges with both endpoints in $V_{r+1}$, in particular $G\left[V_{r+1}\right]$ is complete;

- Given any $1 \leqslant i \leqslant \omega n / b-\eta n$ and $j \leqslant\lceil\sigma i / \omega\rceil$ include all edges $c_{i} a_{j}$.

So at the moment $G$ indeed satisfies the degree sequence in Theorem 7 ; we therefore modify $G$ slightly. For all $k \leqslant i \leqslant k+r b \eta n$ and $\lceil\sigma k / \omega\rceil+1 \leqslant j \leqslant\lceil\sigma(k+$ $r b \eta n) / \omega\rceil$ delete each edge between $c_{i}$ and $a_{j}$. One can easily check that $G$ satisfies the degree sequence in the statement of the proposition. In particular, the vertices of degree $\left(1-\frac{\omega+\sigma}{b}\right) n+\left\lceil\frac{\sigma}{\omega} k\right\rceil+\eta n$ are $c_{k}, \ldots, c_{k+r b \eta n}$.

Define $A:=\left\{a_{1}, \ldots, a_{\lceil\sigma k / \omega\rceil}\right\}$ and $C:=\left\{c_{1}, \ldots, c_{k+r b \eta n}\right\}$. Note that there are no edges between $C$ and $V_{1} \backslash A$ in $G$.

Claim 14. Let $T$ be a $B$-tiling of $G$. Then $T$ does not cover at least $3 \eta n / 2$ vertices in $C$.

Firstly, consider any copy $B^{\prime}$ of $B$ in $T$ that contains at least one vertex in $V_{r+1}$. Since $C$ is an independent set in $G$, observe that $B^{\prime}$ contains at most $\omega$ vertices from $C$. Thus, there are at most $\omega\left|V_{r+1}\right|=\omega(r-1) \eta n+\omega(r-2)$ vertices in $C$ covered by copies of $B$ in $T$ that each contain at least one vertex in $V_{r+1}$.

Secondly, consider any copy $B^{\prime}$ of $B$ in $T$ that contains at least one vertex from $C$ and no vertices from $V_{r+1}$. As before, since $C$ is an independent set in $G$, we have that $B^{\prime}$ contains at most $\omega$ vertices from $C$. Since there are no edges between $C$ and $V_{1} \backslash A$ in $G, B^{\prime}$ contains at least $\sigma$ vertices in $A$.

These two observations, alongside the fact that $b=\sigma+\omega(r-1) \geqslant \omega(r-1) \geqslant \omega(r-2)$, imply that at most $\omega(r-1) \eta n+\omega(r-2)+\lceil\sigma k / \omega\rceil(\omega / \sigma)<k+1+b(\eta n+1)$ vertices in $C$ can be covered by $T$. Since $|C|=k+r b \eta n$, we have that $T$ does not cover at least $3 \eta n / 2$ vertices in $C$. Therefore, Claim 14 holds. Hence $G$ does not have a $B$-tiling covering all but at most $\eta n$ vertices.

Extremal Example 3. Let $H$ be an $h$-vertex graph, $\chi(H)=: r, \sigma:=\sigma(H)$ and $\omega:=(h-\sigma) /(r-1)$. The following extremal example demonstrates that there are $n$-vertex graphs $G$ for which all but $(\omega / h+o(1)) n$ vertices have degree above $\left(1-1 / \chi_{c r}(H)+o(1)\right) n$, with the remaining $(\omega / h+o(1)) n$ vertices having degree $\left(1-1 / \chi_{c r}(H)-o(1)\right) n$, and yet $G$ does not contain a perfect $H$-tiling. Thus, this shows that one cannot have significantly more than $\omega n / h$ 'small' degree vertices in Theorem 7 .

Proposition 15. Let $0<\eta \ll 1$ be fixed. Let $H$ be a graph with $\chi(H)=: r$. Let $h:=|H|$, $\sigma:=\sigma(H)$ and set $\omega:=(h-\sigma) /(r-1)$. Then there exists a graph $G$ on $n$ vertices whose degree sequence $d_{1} \leqslant \cdots \leqslant d_{n}$ satisfies

$$
\begin{gathered}
d_{i}=(1-\omega / h-(r-1) \eta) n=\left(1-1 / \chi_{c r}(H)-(r-1) \eta\right) n \text { for all } i \leqslant(\omega / h+(r-1) \eta) n, \\
d_{i} \geqslant(1-\omega / h+\eta) n=\left(1-1 / \chi_{c r}(H)+\eta\right) n \quad \text { for all } i>(\omega / h+(r-1) \eta) n,
\end{gathered}
$$

but such that $G$ does not contain an $H$-tiling covering all but at most $\eta n$ vertices.

Proof. Let $G$ be the complete $r$-partite graph on $n$ vertices with vertex classes $V_{1}, \ldots, V_{r}$ such that 
- $\left|V_{1}\right|=\frac{\sigma n}{h}-\eta n$,

- $\left|V_{2}\right|=\frac{\omega n}{h}+(r-1) \eta n$,

- $\left|V_{3}\right|=\cdots=\left|V_{r}\right|=\frac{\omega n}{h}-\eta n$.

Then $G$ satisfies the degree sequence condition in the proposition. The choice in size of $V_{1}$ ensures that any $H$-tiling in $G$ covers at most $\left|V_{1}\right| h / \sigma<n-\eta n$ vertices, as desired.

\subsection{A possible strengthening of Theorem 7}

Whilst Proposition 12 demonstrates that we cannot lower every term in the degree sequence condition in Theorem 7 by much, perhaps one can cap the degrees as follows.

Question 16. Can the degree sequence condition in Theorem 7 be replaced by

$$
d_{i} \geqslant \min \left\{\left(1-\frac{\omega+\sigma}{h}\right) n+\frac{\sigma}{\omega} i+\eta n,\left(1-\frac{1}{\chi_{c r}(H)}\right) n+C\right\} \text { for all } 1 \leqslant i \leqslant \frac{\omega n}{h}
$$

where $C$ is a constant dependent only on $H$ ?

Note that Theorem 8 does not quite imply the Kühn-Osthus theorem (Theorem 4) due to the $\eta n$-terms. On the other hand, an affirmative answer to Question 16, together with an analogous 'capped' version of Theorem 8 (ii), would fully imply the upper bound in Theorem 4.

\section{Auxiliary results}

\subsection{The regularity and blow-up lemmas}

The results in this section will be employed in our proof of Theorem 9. First we need the following definitions.

Definition 17. Let $G=(A, B)$ be a bipartite graph with vertex classes $A$ and $B$. We define the density of $G$ to be

$$
d_{G}(A, B):=\frac{e_{G}(A, B)}{|A||B|}
$$

Set $\varepsilon>0$. We say that $G$ is $\varepsilon$-regular if for all $X \subseteq A$ and $Y \subseteq B$ with $|X|>\varepsilon|A|$ and $|Y|>\varepsilon|B|$ we have that $\left|d_{G}(X, Y)-d_{G}(A, B)\right|<\varepsilon$.

Definition 18. Given $\varepsilon>0, d \in[0,1]$ and $G=(A, B)$ a bipartite graph, we say that $G$ is $(\varepsilon, d)$-superregular if all sets $X \subseteq A$ and $Y \subseteq B$ with $|X| \geqslant \varepsilon|A|$ and $|Y| \geqslant \varepsilon|B|$ satisfy that $d(X, Y)>d$, that $d_{G}(a)>d|B|$ for all $a \in A$ and that $d_{G}(b)>d|A|$ for all $b \in B$.

The following groundbreaking result of Szemerédi [19] will be instrumental in our proof of Theorem 9. 
Lemma 19 (Degree form of Szemerédi's Regularity lemma [19]). Let $\varepsilon \in(0,1)$ and $M^{\prime} \in \mathbb{N}$. Then there exist natural numbers $M$ and $n_{0}$ such that for any graph $G$ on $n \geqslant n_{0}$ vertices and any $d \in(0,1)$ there is a partition of the vertices of $G$ into subsets $V_{0}, V_{1}, \ldots, V_{k}$ and a spanning subgraph $G^{\prime}$ of $G$ such that the following hold:

- $M^{\prime} \leqslant k \leqslant M$;

- $\left|V_{0}\right| \leqslant \varepsilon n ;$

- $\left|V_{1}\right|=\cdots=\left|V_{k}\right|=: q$;

- $d_{G^{\prime}}(x)>d_{G}(x)-(d+\varepsilon) n$ for all $x \in V(G)$;

- $e\left(G^{\prime}\left[V_{i}\right]\right)=0$ for all $i \geqslant 1$;

- For all $1 \leqslant i, j \leqslant k$ with $i \neq j$ the pair $\left(V_{i}, V_{j}\right)_{G^{\prime}}$ is $\varepsilon$-regular and has density either 0 or at least $d$.

We call $V_{1}, \ldots, V_{k}$ the clusters of our partition, $V_{0}$ the exceptional set and $G^{\prime}$ the pure graph. We define the reduced graph $R$ of $G$ with parameters $\varepsilon, d$ and $M^{\prime}$ to be the graph whose vertex set is $V_{1}, \ldots, V_{k}$ and in which $V_{i} V_{j}$ is an edge if and only if $\left(V_{i}, V_{j}\right)_{G^{\prime}}$ is $\varepsilon$-regular with density at least $d$. Note also that $|V(R)|=k$.

We will apply the following well known fact, in conjunction with Lemma 21 (below), in Section 7.2.

Fact 20. Let $0<\varepsilon<\alpha$ and $\varepsilon^{\prime}:=\max \{\varepsilon / \alpha, 2 \varepsilon\}$. Let $(A, B)$ be an $\varepsilon$-regular pair of density d. Suppose $A^{\prime} \subseteq A$ and $B^{\prime} \subseteq B$ where $\left|A^{\prime}\right| \geqslant \alpha|A|$ and $\left|B^{\prime}\right| \geqslant \alpha|B|$. Then $\left(A^{\prime}, B^{\prime}\right)$ is an $\varepsilon^{\prime}$-regular pair with density $d^{\prime}$ where $\left|d^{\prime}-d\right|<\varepsilon$.

Lemma 21 (Key lemma [13]). Suppose that $0<\varepsilon<d$, that $q, t \in \mathbb{N}$ and that $R$ is a graph with $V(R)=\left\{v_{1}, \ldots, v_{k}\right\}$. We construct a graph $G$ as follows: Replace every vertex $v_{i} \in V(R)$ with a set $V_{i}$ of $q$ vertices and replace each edge of $R$ with an $\varepsilon$-regular pair of density at least $d$. For each $v_{i} \in V(R)$, let $U_{i}$ denote the set of $t$ vertices in $R(t)$ corresponding to $v_{i}$. Let $H$ be a subgraph of $R(t)$ with maximum degree $\Delta$ and set $h:=|H|$. Set $\delta:=d-\varepsilon$ and $\varepsilon_{0}:=\delta^{\Delta} /(2+\Delta)$. If $\varepsilon \leqslant \varepsilon_{0}$ and $t-1 \leqslant \varepsilon_{0} q$ then there are at least

\section{$\left(\varepsilon_{0} q\right)^{h}$ labelled copies of $H$ in $G$}

so that if $x \in V(H)$ lies in $U_{i}$ in $R(t)$, then $x$ is embedded into $V_{i}$ in $G$.

Let $G$ be a graph as in Theorem 9 and $R$ a reduced graph of $G$. The next well known lemma essentially says that $R$ 'inherits' the degree sequence of $G$.

Lemma 22 (See e.g. [7]). Set $M^{\prime}, n_{0} \in \mathbb{N}$ and $\varepsilon, d, \eta, b, \omega, \sigma$ to be positive constants such that $1 / n_{0} \ll 1 / M^{\prime} \ll \varepsilon \ll d \ll \eta, 1 / b$ and where $\omega+\sigma \leqslant b$. Suppose $G$ is a graph on $n \geqslant n_{0}$ vertices with degree sequence $d_{1} \leqslant \cdots \leqslant d_{n}$ such that

$$
d_{i} \geqslant \frac{b-\omega-\sigma}{b} n+\frac{\sigma}{\omega} i+\eta n \text { for all } 1 \leqslant i \leqslant \frac{\omega n}{b} .
$$


Let $R$ be the reduced graph of $G$ with parameters $\varepsilon, M^{\prime}$ and $d$ and set $k:=|V(R)|$. Then $R$ has degree sequence $d_{R, 1} \leqslant \cdots \leqslant d_{R, k}$ such that

$$
d_{R, i} \geqslant \frac{b-\omega-\sigma}{b} k+\frac{\sigma}{\omega} i+\frac{\eta k}{2} \text { for all } 1 \leqslant i \leqslant \frac{\omega k}{b} .
$$

Let $G$ and $H$ be graphs and $R$ be a reduced graph of $G$. Let $\mathcal{H}$ be a perfect $H$-tiling in $R$. The following result ensures that after removing only a few vertices from each cluster in $R$ every edge in each copy of $H \in \mathcal{H}$ corresponds to a superregular pair. This will be essential to apply Lemma 24 in Section 7.4.

Proposition 23 (See e.g. [16]). Let $G$ be a graph, $\varepsilon, d \in(0,1)$ and $M^{\prime}, \Delta \in \mathbb{N}$. Apply Lemma 19 to $G$ with parameters $\varepsilon, M^{\prime}$ and $d$ to obtain a reduced graph $R$ with clusters of size $q$. Let $H$ be a subgraph of the reduced graph $R$ with $\Delta(H) \leqslant \Delta$ and label the vertices of $H$ as $V_{1}, \ldots, V_{|H|}$. Then each vertex $V_{i}$ of $H$ contains a subset $V_{i}^{\prime}$ of size $(1-\varepsilon \Delta) q$ such that for every edge $V_{i} V_{j}$ of $H$ the graph $\left(V_{i}^{\prime}, V_{j}^{\prime}\right)_{G^{\prime}}$ is $(\varepsilon /(1-\varepsilon \Delta), d-(1+\Delta) \varepsilon)$-superregular.

The following fundamental result of Komlós, Sárközy and Szemerédi [11], known as the Blow-up lemma, essentially says that $(\varepsilon, d)$-superregular pairs behave like complete bipartite graphs with respect to containing bounded degree subgraphs.

Lemma 24 (Blow-up lemma [11])). Given a graph $F$ on vertices $\{1, \ldots, f\}$ and $d, \Delta>0$, there exists an $\varepsilon_{0}=\varepsilon_{0}(d, \Delta, f)>0$ such that the following holds. Given $L_{1}, \ldots, L_{f} \in \mathbb{N}$ and $\varepsilon \leqslant \varepsilon_{0}$, let $F^{*}$ be the graph obtained from $F$ by replacing each vertex $i \in F$ with a set $V_{i}$ of $L_{i}$ new vertices and joining all vertices in $V_{i}$ to all vertices in $V_{j}$ whenever ij is an edge of $F$. Let $G$ be a spanning subgraph of $F^{*}$ such that for every edge ij $\in F$ the pair $\left(V_{i}, V_{j}\right)_{G}$ is $(\varepsilon, d)$-superregular. Then $G$ contains a copy of every subgraph $H$ of $F^{*}$ with $\Delta(H) \leqslant \Delta$.

\subsection{Tilings in complete graphs}

In [14], the following result of Kühn and Osthus is essential to their proof of Theorem 5 .

Lemma 25. [15, Lemma 12] Let $H$ be a graph with $\chi(H)=: r \geqslant 2$ and $\operatorname{hcf}(H)=1$. Let $h:=|H|$ and $\omega(H):=(h-\sigma(H)) /(r-1)$. Let $0<\beta_{1} \ll \lambda_{1} \ll \sigma(H) / \omega(H)$, $1-\sigma(H) / \omega(H), 1 / h$ be positive constants. Suppose that $F$ is a complete $r$-partite graph with vertex classes $U_{1}, \ldots, U_{r}$ such that: $1 /|F| \ll \beta_{1}$;

$|F|$ is divisible by $h ;\left(1-\lambda_{1}^{1 / 10}\right)\left|U_{r}\right| \leqslant \sigma(H)\left|U_{i}\right| / \omega(H) \leqslant\left(1-\lambda_{1}\right)\left|U_{r}\right|$ for all $i<r ;$ ||$U_{i}|-| U_{j}|| \leqslant \beta_{1}|F|$ whenever $1 \leqslant i<j<r$. Then $F$ contains a perfect $H$-tiling.

We will use the Blow-up lemma in tandem with the following generalisation of Lemma $25^{1}$ to conclude that a particular tiling that we construct in a reduced graph $R$ guarantees a perfect $H$-tiling in our original graph $G$.

\footnotetext{
${ }^{1}$ Note that Lemma 25 is the $\sigma=\sigma(H)$ case of Lemma 26.
} 
Lemma 26. Let $H$ be a graph with $\chi(H)=: r \geqslant 2$ and $\operatorname{hcf}(H)=1$. Let $h:=|H|$. Set $\sigma \in \mathbb{R}$ such that $\sigma(H) \leqslant \sigma<h / r$ and $\omega:=(h-\sigma) /(r-1)$. Let $0<\beta_{2} \ll \lambda_{2} \ll \sigma / \omega$, $1-\sigma / \omega, 1 / h$ be positive constants. Suppose that $F$ is a complete $r$-partite graph with vertex classes $U_{1}, \ldots, U_{r}$ such that: $1 /|F| \ll \beta_{2} ;|F|$ is divisible by $h ;\left(1-\lambda_{2}^{1 / 10}\right)\left|U_{r}\right| \leqslant$ $\sigma\left|U_{i}\right| / \omega \leqslant\left(1-\lambda_{2}\right)\left|U_{r}\right|$ for all $i<r ;|| U_{i}|-| U_{j}|| \leqslant \beta_{2}|F|$ whenever $1 \leqslant i<j<r$. Then $F$ contains a perfect $H$-tiling.

Proof. Note we may assume that $\sigma>\sigma(H)$ as otherwise the result follows immediately from Lemma 25. We choose $\beta_{2} \ll \beta_{1} \ll \lambda_{2} \ll \lambda_{1}$ where $\beta_{1}$ and $\lambda_{1}$ are as in Lemma 25 . Additionally we may assume $\beta_{2}, \lambda_{2} \ll(\sigma / \omega-\sigma(H) / \omega(H))$.

Let $F$ be as in the statement of the lemma. Set $H^{*}$ to be the complete balanced $r$-partite graph on $r h$ vertices (that is, each vertex class of $H^{*}$ has size $h$ ). Observe that $H^{*}$ has a perfect $H$-tiling using precisely $r$ copies of $H$.

Repeatedly delete disjoint copies of $H^{*}$ from $F$ (and therefore update the classes $\left.U_{1}, \ldots, U_{r}\right)$ until the first point for which there is some $i<r$ such that

$$
\left(1-\lambda_{1}^{1 / 10} / 2\right)\left|U_{r}\right| \leqslant \sigma(H)\left|U_{i}\right| / \omega(H) \leqslant\left(1-2 \lambda_{1}\right)\left|U_{r}\right|
$$

Call the resulting graph $F^{\prime}$. Note that $\sigma / \omega>\sigma(H) / \omega(H)$, so we can indeed obtain $F^{\prime}$. Further note that our (sufficiently small) choice of $\beta_{2}$ ensures each class $U_{j}$ still contains at least a $\beta_{2}^{1 / 2}$-proportion of the vertices it started with. So now ||$U_{i}|-| U_{j}|| \leqslant \beta_{2}|F| \leqslant$ $\beta_{2}^{1 / 2}\left|F^{\prime}\right| \leqslant \beta_{1}\left|F^{\prime}\right|$ whenever $1 \leqslant i<j<r$. Moreover, this implies

$$
\left(1-\lambda_{1}^{1 / 10}\right)\left|U_{r}\right| \leqslant \sigma(H)\left|U_{j}\right| / \omega(H) \leqslant\left(1-\lambda_{1}\right)\left|U_{r}\right|
$$

for all $j<r$. Thus, by Lemma $25, F^{\prime}$ contains a perfect $H$-tiling and therefore, so too does $F$, as desired.

\subsection{A degree sequence Komlós theorem}

In [14], Kühn and Osthus begin their proof of Theorem 5 by applying Komlós' theorem (Theorem 3).

In our proof of Theorem 9 we will use the following degree sequence version of Komlós' theorem that the authors and Liu proved in [7].

Theorem 27. [7, Theorem 8.1] Let $\eta>0$ and $H$ be a graph with $\chi(H)=: r$ and $h:=|H|$. Set $\sigma \in \mathbb{R}$ such that $\sigma(H) \leqslant \sigma \leqslant h / r$ and $\omega:=(h-\sigma) /(r-1)$. Then there exists an integer $n_{0}=n_{0}(\eta, \sigma, H) \in \mathbb{N}$ such that the following holds. Suppose $G$ is a graph on $n \geqslant n_{0}$ vertices with degree sequence $d_{1} \leqslant \cdots \leqslant d_{n}$ such that

$$
d_{i} \geqslant\left(1-\frac{\omega+\sigma}{h}\right) n+\frac{\sigma}{\omega} i \text { for all } 1 \leqslant i \leqslant \frac{\omega n}{h} .
$$

Then $G$ contains an $H$-tiling covering all but at most $\eta n$ vertices. 


\subsection{Bézout's Lemma}

To prove Theorem 30 we will need the following elementary arithmetic result.

Lemma 28 (Bézout's Lemma). Let $a_{1}, a_{2}, \ldots, a_{t} \in \mathbb{Z}$. Then there exist integers $y_{1}, y_{2}, \ldots, y_{t} \in \mathbb{Z}$ such that

$$
\sum_{i=1}^{t} y_{i} a_{i}=\operatorname{hcf}\left(a_{1}, a_{2}, \ldots, a_{t}\right)
$$

where $\operatorname{hcf}\left(a_{1}, a_{2}, \ldots, a_{t}\right)$ is the highest common factor of $a_{1}, a_{2}, \ldots, a_{t}$.

\section{A tool for the proof of Theorem 9}

In this section, we prove a theorem (Theorem 30) that will be used in Sections 7.3.1 and 7.3.2 of the proof of Theorem 9. At the beginning of Section 7.3, we will have a certain $\hat{B}$-tiling $\hat{\mathcal{B}}$ of a reduced graph $R$ (the graph $\hat{B}$ will be defined later). Denote the copies of $\hat{B}$ in $\hat{\mathcal{B}}$ by $\hat{B}_{1}, \hat{B}_{2}, \ldots, \hat{B}_{\hat{k}}$. For applications of Lemma 26 required at the end of our proof of Theorem 9, we will need $\left|V_{G}\left(\hat{B}_{i}\right)\right|$ to be divisible by $h$ for each $1 \leqslant i \leqslant \hat{k}$. The following theorem is the crucial tool for ensuring we can remove copies of $H$ from $G$ to achieve this.

For a graph $H$ with $\chi(H)=r$, recall that $C_{H}$ is the set of all optimal colourings of $H$ and that given an optimal colouring $c \in C_{H}$ we let $x_{c, 1} \leqslant x_{c, 2} \leqslant \ldots \leqslant x_{c, r}$ denote the sizes of the colour classes of $c$. We require the following definitions.

Definition 29. Let $H$ be a graph with $\chi(H)=: r$. Fix $1 \leqslant p \leqslant r-1$. For each $c \in C_{H}$, define $D_{c}$ to be the multiset $\left[x_{c, 1}, x_{c, 2}, \ldots, x_{c, r}\right]$. We say that $A$ is a $p$-subset contained in $D_{c}$ if $A$ is a multiset, $|A|=p$ and $A=\left[x_{c, j_{1}}, x_{c, j_{2}}, \ldots, x_{c, j_{p}}\right]$ where $j_{1}, j_{2}, \ldots, j_{p} \in\{1, \ldots, r\}$ are distinct. Let $z_{p}:=\left(\begin{array}{l}r \\ p\end{array}\right)$ be the number of $p$-subsets contained in $D_{c}$. For each colouring $c \in C_{H}$, label the $p$-subsets contained in $D_{c}$ by $A_{p, c, 1}, A_{p, c, 2}, \ldots, A_{p, c, z_{p}}$. Let $S_{p, c, J}:=\sum_{x \in A_{p, c, J}} x$ for each $c \in C_{H}, 1 \leqslant J \leqslant z_{p}$.

Theorem 30. Let $H$ be an $r$-partite graph and let $h:=|H|$. Fix $1 \leqslant p \leqslant r-1$. Let $b$ be the number of components of $H$ and $t_{1}, \ldots, t_{b}$ be the sizes of the components of $H$. Then

- if $r=2$ and $\operatorname{hcf}_{c}(H)=1$, there exists a collection of non-negative integers $\left\{a_{i}: 1 \leqslant\right.$ $i \leqslant b\}$ and $\bar{a} \in \mathbb{N}$ such that

$$
a_{i} \leqslant \bar{a} \text { for all } 1 \leqslant i \leqslant b
$$

and

$$
\sum_{i=1}^{b} a_{i} t_{i} \equiv 1 \quad \bmod h
$$


- if $r \geqslant 3$ and $\operatorname{hcf}_{\chi}(H)=1$, there exists a collection of non-negative integers $\left\{a_{p, c, i}\right.$ : $\left.c \in C_{H}, 1 \leqslant i \leqslant z_{p}\right\}$ and $\bar{a} \in \mathbb{N}$ such that

$$
a_{p, c, i} \leqslant \bar{a} \text { for all } c \in C_{H} \text { and } 1 \leqslant i \leqslant z_{p}
$$

and

$$
\sum_{c \in C_{H}} \sum_{i=1}^{z_{p}} a_{p, c, i} S_{p, c, i} \equiv 1 \quad \bmod h .
$$

For each $1 \leqslant p \leqslant r-1, c \in C_{H}$ and $j \in\{1, \ldots, r\}$, let $Z_{p, c, j}$ be the multiset defined by the following table:

\begin{tabular}{c|c|c|c|c|c|c|c} 
Colour class size & $x_{c, 1}$ & $\cdots$ & $x_{c, j-1}$ & $x_{c, j}$ & $x_{c, j+1}$ & $\cdots$ & $x_{c, r}$ \\
\hline Multiplicity in $Z_{p, c, j}$ & $p$ & $\cdots$ & $p$ & $p+1$ & $p$ & $\cdots$ & $p$
\end{tabular}

The following fact will be useful in our proof of Theorem 30 .

Fact 31. For any $1 \leqslant J, L \leqslant r$, we can partition $Z_{p, c, J}$ into $\left\{x_{c, L}\right\}$ and $r$ p-subsets contained in $D_{c}$.

Proof of Theorem 30. Firstly, we will consider the case when $r=2$ and $\operatorname{hcf}_{c}(H)=1$. So $H$ must have multiple components. The sizes of these components of $H$ are $t_{1}, t_{2}, \ldots, t_{b}$. Since $\operatorname{hcf}_{c}(H)=1$, by Bezout's Lemma (Lemma 28) there exist integers $a_{1}^{\prime}, \ldots, a_{b}^{\prime}$ such that

$$
\sum_{i=1}^{b} a_{i}^{\prime} t_{i}=\operatorname{hcf}\left(t_{1}, \ldots, t_{b}\right)=1 .
$$

Since $\sum_{i=1}^{b} t_{i}=h$, there exists $\hat{a} \in \mathbb{N} \cup\{0\}$ such that

$$
\sum_{i=1}^{b}\left(a_{i}^{\prime}+\hat{a}\right) t_{i} \equiv 1 \quad \bmod h
$$

and

$$
a_{i}^{\prime}+\hat{a} \geqslant 0 \text { for all } 1 \leqslant i \leqslant b .
$$

For each $1 \leqslant i \leqslant b$, take $a_{i}:=a_{i}^{\prime}+\hat{a}$ and $\bar{a}:=\max _{i=1, \ldots, b} a_{i}$.

Next consider when $r \geqslant 3$. Instead of explicitly calculating $a_{p, c, i}$ for each $c \in C_{H}$, $1 \leqslant i \leqslant z_{p}$, we will construct for each $c \in C_{H}$ a multiset $X_{c}$ of bounded size which can be partitioned into $p$-subsets contained in $D_{c}$. Further, we will construct our multisets $X_{c}$ such that

$$
\sum_{c \in C_{H}} \sum_{x \in X_{c}} x \equiv 1 \bmod h
$$

Observe that constructing such multisets $X_{c}$ immediately yields a collection of nonnegative integers $\left\{a_{p, c, i}: c \in C_{H}, 1 \leqslant i \leqslant z_{p}\right\}$ that satisfy the conditions in Theorem 30 . 
Indeed, for each $c \in C_{H}$ and $1 \leqslant i \leqslant z_{p}$, we take $a_{p, c, i}$ to be precisely the number of times $A_{p, c, i}$ occurs in the partition of $X_{c}$ into $p$-subsets.

In order to start constructing our multisets $X_{c}$, we define the following multiset:

$$
\mathcal{D}^{*}(H):=\bigcup_{c \in C_{H}}\left[x_{c, j+1}-x_{c, j} \mid j=1, \ldots, r-1\right] .
$$

As $\operatorname{hcf}_{\chi}(H)=1$ we can apply Lemma 28 to the multiset $\mathcal{D}^{*}(H)$ to get for each $c \in C_{H}$, $1 \leqslant j \leqslant r-1$ integers $b_{c, j}$ such that the following holds:

$$
\sum_{c \in C_{H}} \sum_{j=1}^{r-1} b_{c, j}\left(x_{c, j+1}-x_{c, j}\right) \equiv 1 \quad \bmod h .
$$

We now construct our multisets $X_{c}$. Fix $c \in C_{H}$. Choose $t_{c} \in \mathbb{N}$ to be the smallest natural number such that

$$
p t_{c} \geqslant \max \left\{\left|b_{c, 1}\right|,\left|b_{c, 1}-b_{c, 2}\right|,\left|b_{c, 2}-b_{c, 3}\right|, \ldots,\left|b_{c, r-2}-b_{c, r-1}\right|,\left|b_{c, r-1}\right|\right\} .
$$

Then $p t_{c}-b_{c, 1}, p t_{c}+b_{c, 1}-b_{c, 2}, p t_{c}+b_{c, 2}-b_{c, 3}, \ldots, p t_{c}+b_{c, r-2}-b_{c, r-1}, p t_{c}+b_{c, r-1}$ are non-negative integers. Let $Y_{c}$ be the multiset defined by the following table:

\begin{tabular}{l|l|l|l|l|l|l}
$\begin{array}{l}\text { Colour } \\
\text { class size }\end{array}$ & $x_{c, 1}$ & $x_{c, 2}$ & $x_{c, 3}$ & $\ldots$ & $x_{c, r-1}$ & $x_{c, r}$ \\
\hline $\begin{array}{l}\text { Multiplicity } \\
\text { in } Y_{c}\end{array}$ & $p t_{c}-b_{c, 1}$ & $\begin{array}{l}p t_{c}+b_{c, 1}- \\
b_{c, 2}\end{array}$ & $\begin{array}{l}p t_{c}+b_{c, 2}- \\
b_{c, 3}\end{array}$ & $\ldots$ & $p t_{c}+b_{c, r-2}-$ & $p t_{c}+b_{c, r-1}$ \\
\end{tabular}

Then $\left|Y_{c}\right|=r p t_{c}$. If we can partition $Y_{c}$ into $p$-subsets contained in $D_{c}$ then we take $X_{c}:=Y_{c}$. Assume we cannot. Then the multiplicities of $x_{c, 1}, \ldots, x_{c, r}$ in $Y_{c}$ must be sufficiently different from one another. We employ the following algorithm which transforms $Y_{c}$ into a multiset which can be partitioned into $p$-subsets contained in $D_{c}$ using Fact 31. To state the algorithm we require the following definition.

Definition 32. For each $c \in C_{H}, 1 \leqslant i \leqslant r$, let $m_{c, i}$ be the multiplicity of $x_{c, i}$ in $Y_{c}$. Let $J, L \in\{1, \ldots, r\}$ such that $J \neq L ; m_{c, J} \geqslant \frac{\sum_{i=1}^{r} m_{c, i}}{r} ; m_{c, L} \leqslant \frac{\sum_{i=1}^{r} m_{c, i}}{r} ; m_{c, L}+1 \neq m_{c, J}$; $m_{c, L} \neq m_{c, J}$. Let $Y_{c}^{\prime}:=Y_{c}-\left\{x_{c, J}\right\}+\left\{x_{c, L}\right\} .^{2}$ Then we say that $Y_{c}^{\prime}$ is more balanced than $Y_{c}$.

\section{Algorithm.}

1) Let $Q:=\emptyset$.

2) If $\left|m_{c, i}-m_{c, j}\right|=0$ for all $1 \leqslant i, j \leqslant r$, output $Y_{c}$ and $Q$. Otherwise, choose $J, L \in\{1, \ldots, r\}$ such that $Y_{c}^{\prime}:=Y_{c}-\left\{x_{c, J}\right\}+\left\{x_{c, L}\right\}$ is more balanced than $Y_{c}$.

\footnotetext{
${ }^{2}$ That is, $Y_{c}^{\prime}$ is the multiset $Y_{c}$ except with $x_{c, J}$ having multiplicity $m_{c, J}-1$ and $x_{c, L}$ having multiplicity $m_{c, L}+1$.
} 
3) Add $p$ copies of $H$ with colouring $c$ to $Y_{c}$. That is, $x_{c, i}$ now has multiplicity $m_{c, i}+p$ in $Y_{c}$ for each $1 \leqslant i \leqslant r$.

4) Take $Z_{p, c, J}$ to be the union of $\left\{x_{c, J}\right\}$ and these $p$ copies of $H$. By Fact 31 there exists a partition of $Z_{p, c, J}$ into $\left\{x_{c, L}\right\}$ and $r p$-subsets contained in $D_{c}$.

5) Place into $Q$ these $r p$-subsets contained in $D_{c}$.

6) Take $Y_{c}:=Y_{c}^{\prime}$ and update the value of each $m_{c, i}$ (that is, $m_{c, J}$ has decreased by 1 and $m_{c, L}$ has increased by 1). Go to Step 2.

Therefore, at the end of the algorithm $\left|Y_{c}\right|=r p t_{c}$ and $\left|m_{c, i}-m_{c, j}\right|=0$ for all $1 \leqslant$ $i, j \leqslant r$. In particular, it is easy to see that $Y_{c}$ now has a partition $Q_{Y_{c}}$ into $p$-subsets contained in $D_{c}$. Let $t_{c}^{\prime}$ be the number of collections of $p$ copies of $H$ added during the algorithm and $\hat{t}_{c}:=t_{c}+t_{c}^{\prime}$. Then the multiset $\hat{Y}_{c}$, defined by the table below, can be partitioned into $p$-subsets contained in $D_{c}$ using the partition $Q \cup Q_{Y_{c}}$ :

\begin{tabular}{l|l|l|l|l|l|l}
$\begin{array}{l}\text { Colour } \\
\text { class size }\end{array}$ & $x_{c, 1}$ & $x_{c, 2}$ & $x_{c, 3}$ & $\ldots$ & $x_{c, r-1}$ & $x_{c, r}$ \\
\hline $\begin{array}{l}\text { Multiplicity } \\
\text { in } \hat{Y}_{c}\end{array}$ & $p \hat{t}_{c}-b_{c, 1}$ & $p \hat{t}_{c}+b_{c, 1}-$ & $p \hat{t}_{c}+b_{c, 2}-$ & $\ldots$ & $p \hat{t}_{c}+b_{c, r-2}-$ & $p \hat{t}_{c}+$ \\
$b_{c, 2}$
\end{tabular}

Take $X_{c}:=\hat{Y}_{c}$. We now confirm that our multisets $X_{c}$ satisfy

$$
\sum_{c \in C_{H}} \sum_{x \in X_{c}} x \equiv 1 \bmod h
$$

By (1) and the definition of $X_{c}$ for each $c \in C_{H}$ we have

$$
\begin{aligned}
& \sum_{c \in C_{H}} \sum_{x \in X_{c}} x \\
= & \sum_{c \in C_{H}}\left(\sum_{j=1}^{r-1} b_{c, j}\left(x_{c, j+1}-x_{c, j}\right)+p \hat{t}_{c}\left(\sum_{j=1}^{r} x_{c, j}\right)\right) \\
= & \left(\sum_{c \in C_{H}} \sum_{j=1}^{r-1} b_{c, j}\left(x_{c, j+1}-x_{c, j}\right)\right)+\left(p \sum_{c \in C_{H}} \hat{t}_{c}\right) h \\
\stackrel{(1)}{\equiv} & 1 \bmod h .
\end{aligned}
$$

Therefore, recalling the discussion earlier in this proof, there must exist the desired collection of non-negative integers $\left\{a_{p, c, i}: c \in C_{H}, 1 \leqslant i \leqslant z_{p}\right\}$, and we take $\bar{a}$ to be the maximum element in this collection. 


\section{Proof Overview}

The rest of this paper will be devoted to proving Theorem 9 and here we outline the proof. As noted in Section 1.2, our proof follows closely Kühn and Osthus' proof of Theorem 5 in $[14]$.

Let $H, G, \eta$ and $\sigma$ be as in the statement of the theorem. In particular, $h:=|H|$ and $\omega:=(h-\sigma) /(r-1)$. Note that it suffices to prove the result in the case when $\sigma \in \mathbb{Q}$. First we define a bottle graph $B$ that contains a perfect $H$-tiling.

Definition 33. Let $a, b \in \mathbb{N}$ such that $\sigma=a / b$. Let $\omega(H):=(h-\sigma(H)) /(r-1)$ and $\hat{c}:=b(r-1)(\omega(H)-\sigma(H))$. Define $B$ to be the $r$-partite bottle graph with neck $\sigma \hat{c}$ and width $\omega \hat{c}$ (note that $\sigma \hat{c}, \omega \hat{c} \in \mathbb{N}$ ). Observe that $|B|=h \hat{c} ; \sigma(B)=\sigma \hat{c} ; \omega(B)=\omega \hat{c}$. Further,

$$
\chi_{c r}(B)=r-1+\sigma / \omega=h / \omega .
$$

Since $|B|=h \hat{c} ; \sigma(B)=\sigma \hat{c} ; \omega(B)=\omega \hat{c}$, we have that $G$ satisfies the hypothesis of the degree sequence Komlós theorem (Theorem 27) with $B, \sigma(B)$ and $\omega(B)$ playing the roles of $H, \sigma$ and $\omega$ respectively. That is, $G$ contains an almost perfect $B$-tiling. In fact, as the reduced graph $R$ of $G$ almost inherits the degree sequence of $G$, Theorem 27 ensures that $R$ contains an almost perfect $B$-tiling $\mathcal{B}$. Further note that the choice of $\hat{c}$ implies that $B$ has a perfect $H$-tiling consisting of $\hat{c}$ copies of $H$. Indeed, this follows as $B$ has a perfect tiling of $a-\sigma(H) b$ copies of $K_{h, \ldots, h}$ and $h b-a r$ copies of $H$, where $K_{h, \ldots, h}$ is the complete $r$-partite graph with each vertex class having size $h$.

Ideally one would like to use $\mathcal{B}$ as a framework to build the perfect $H$-tiling in $G$. However, as explained shortly, we need more flexibility in our tiling in $R$. Therefore, we introduce the following 'modified' version of $B$.

Definition 34. Let $s \in \mathbb{N}$ be sufficiently large and $\lambda \in \mathbb{R}^{+}$be sufficiently small where $\sigma(1+\lambda) s / \omega \in \mathbb{N}$. Recall that $\sigma<\omega$. Let $\hat{B}$ be the $r$-partite bottle graph with neck $\sigma(1+\lambda) s / \omega$ and width $s .{ }^{3}$ Moreover, we choose $\lambda$ and $s$ such that $\hat{B}$ contains a perfect $B$-tiling. Hence $\hat{B}$ contains a perfect $H$-tiling. Note that

$$
\chi_{c r}(\hat{B})=r-1+\sigma(1+\lambda) / \omega .
$$

Since $\lambda$ is chosen to be small (and so $\chi_{c r}(\hat{B})$ is very close to $\chi_{c r}(B)$ ), one can still apply Theorem 27 on inputs $\hat{B}$ and $R$. That is, $R$ contains an almost perfect $\hat{B}$-tiling $\hat{\mathcal{B}}$. Denote the copies of $\hat{B}$ in $\hat{\mathcal{B}}$ by $\hat{B}_{1}, \hat{B}_{2}, \ldots, \hat{B}_{\hat{k}}$. By removing a small number of vertices from each cluster in $R$, we can ensure the edges of each $\hat{B}_{i}$ correspond to superregular pairs. Let $V_{0}$ denote the set of all vertices in $G$ not contained in the clusters lying in the tiling $\hat{\mathcal{B}}$.

For each $1 \leqslant i \leqslant \hat{k}$, let $\hat{G}_{i}$ be the $r$-partite subgraph of $G$ whose $j$ th vertex class is the union of all those clusters contained in the $j$ th vertex class of $\hat{B}_{i}$, for each $1 \leqslant j \leqslant r$. Let $G_{i}^{*}$ be the complete $r$-partite graph on the same vertex set as $\hat{G}_{i}$. We introduce the graph $\hat{B}$ (rather than just working with $B$ ) since $\hat{B}$ has the following crucial property:

\footnotetext{
${ }^{3}$ We have that $\sigma(1+\lambda) / \omega<1$ by our choice of $\lambda$ and that $\sigma<\omega$.
} 
For each $1 \leqslant i \leqslant \hat{k}$ we can arbitrarily delete a small number of vertices from $G_{i}^{*}$ (and correspondingly $\hat{G}_{i}$ ) and, provided $\left|V\left(G_{i}^{*}\right)\right|$ is now divisible by $h$, the resulting graph satisfies the hypothesis of Lemma 26. That is, this graph contains a perfect $H$-tiling. Then the Blow-up lemma (Lemma 24) implies that each $\hat{G}_{i}$ contains a perfect $H$-tiling.

We make use of this property of $\hat{B}$ as follows: In Section 7.2 we remove copies of $H$ from $G$ that cover all vertices in $V_{0}$, as well as a small (possibly zero) number of vertices from each $\hat{G}_{i}$; call this $H$-tiling (formed from these copies of $H$ ) $\mathcal{H}_{1}$. Deleting these covered vertices from each $\hat{G}_{i}$, if $\left|V\left(\hat{G}_{i}\right)\right|\left(=\left|V\left(G_{i}^{*}\right)\right|\right)$ is still divisible by $h$ for each $1 \leqslant i \leqslant \hat{k}$ then each $\hat{G}_{i}$ now contains a perfect $H$-tiling (by our argument above). However, for some $i$, we may have that $\left|V\left(\hat{G}_{i}\right)\right|$ is not divisible by $h$. So in Section 7.3 we remove a further bounded number of copies of $H$, forming an $H$-tiling $\mathcal{H}_{2}$, to ensure $\left|V\left(\hat{G}_{i}\right)\right|\left(=\left|V\left(G_{i}^{*}\right)\right|\right)$ is divisible by $h$ for each $1 \leqslant i \leqslant \hat{k}$. Thus, we now have that each $\hat{G}_{i}$ contains a perfect $H$-tiling $\hat{\mathcal{H}}_{i}$. Combining the tilings $\mathcal{H}_{1}, \mathcal{H}_{2}, \hat{\mathcal{H}}_{1}, \ldots, \hat{\mathcal{H}}_{\hat{k}}$ yields a perfect $H$-tiling in $G$, as desired.

Our argument in Section 7.3 will split into two cases, the first being when $\chi(H) \geqslant 3$ and the latter when $H$ is bipartite. This is where our proof differs the most from that in [14] as we must make use of Theorem 30 to find suitable copies of $H$ to ensure each $\left|V\left(\hat{G}_{i}\right)\right|$ is divisible by $h$.

\section{$7 \quad$ Proof of Theorem 9}

\subsection{Applying the regularity lemma}

Note that it suffices to prove the theorem in the case when $\sigma \in \mathbb{Q}$. Let $n$ be sufficiently large and fix constants that satisfy the following hierarchy

$$
0<1 / n \ll 1 / M^{\prime} \ll \varepsilon \ll d \ll \eta_{1} \ll \beta \ll \alpha \ll \lambda \ll \eta, \sigma / \omega, 1-\sigma / \omega, 1 / h .
$$

As discussed in Section 6, we choose $s \in \mathbb{N}$ sufficiently large and define $\hat{B}$ to be the $r$-partite bottle graph with neck $\sigma(1+\lambda) s / \omega$ and width $s$. As before, we choose $\lambda$ and $s$ such that $\hat{B}$ contains a perfect $B$-tiling, which implies that $\hat{B}$ contains a perfect $H$-tiling. Note again that

$$
\chi_{c r}(\hat{B})=r-1+\sigma(1+\lambda) / \omega .
$$

Moreover, choose $\eta_{1}$ and $M^{\prime}$ such that

$$
\eta_{1} \ll 1 /|\hat{B}| \text { and } M^{\prime} \geqslant n_{0}\left(\eta_{1}, \sigma(\hat{B}), \hat{B}\right),
$$

where $n_{0}$ is defined as in Theorem 27. Let $G$ be an $n$-vertex graph as in the statement of Theorem 9. Apply Lemma 19 with parameters $\varepsilon, d$ and $M^{\prime}$ to $G$ to obtain clusters $V_{1}, \ldots, V_{k}$, an exceptional set $V_{0}$ and a pure graph $G^{\prime}$, where $q:=\left|V_{1}\right|=\cdots=\left|V_{k}\right|$ and $k \geqslant M^{\prime}$. Let $R$ be the corresponding reduced graph. Using (2), we may apply Lemma 22 with parameters $M^{\prime}, n, \varepsilon, d, \eta, h, \omega, \sigma$ to conclude that $R$ has degree sequence $d_{R, 1} \leqslant d_{R, 2} \leqslant \cdots \leqslant d_{R, k}$ where

$$
d_{R, i} \geqslant\left(1-\frac{\omega+\sigma}{h}\right) k+\frac{\sigma}{\omega} i+\frac{\eta k}{2} \text { for all } 1 \leqslant i \leqslant \frac{\omega k}{h} .
$$


For a graph $F$, recall that $\sigma(F)$ denotes the size of the smallest possible colour class in any $\chi(F)$-colouring of $F$ and $\omega(F):=(|F|-\sigma(F)) /(\chi(F)-1)$. Since $\lambda \ll \eta$, we have that

$$
d_{R, i} \geqslant\left(1-\frac{\omega(\hat{B})+\sigma(\hat{B})}{|\hat{B}|}\right) k+\frac{\sigma(\hat{B})}{\omega(\hat{B})} i \text { for all } 1 \leqslant i \leqslant \frac{\omega(\hat{B}) k}{|\hat{B}|} .
$$

Since $|V(R)|=k \geqslant M^{\prime} \geqslant n_{0}\left(\eta_{1}, \sigma(\hat{B}), \hat{B}\right)$ and (4) holds, we apply Theorem 27 to find a $\hat{B}$-tiling $\hat{\mathcal{B}}$ covering all but at most $\eta_{1} k$ vertices in $R$. Denote the copies of $\hat{B}$ in $\hat{\mathcal{B}}$ by $\hat{B}_{1}, \hat{B}_{2}, \ldots, \hat{B}_{\hat{k}}$. Now delete all clusters not contained in some $\hat{B}_{i}$ from $R$ and add the vertices in these clusters to $V_{0}$. Therefore now

$$
\left|V_{0}\right| \leqslant \varepsilon n+\eta_{1} n \leqslant 2 \eta_{1} n
$$

From now on, we denote by $R$ the subgraph of the reduced graph induced by all the remaining clusters and redefine $k:=|V(R)|$. Since $\eta_{1} \ll \eta$, (3) implies that $R$ has degree sequence $d_{R, 1} \leqslant d_{R, 2} \leqslant \cdots \leqslant d_{R, k}$ where

$$
d_{R, i} \geqslant\left(1-\frac{\omega+\sigma}{h}\right) k+\frac{\sigma}{\omega} i+\frac{\eta k}{4} \text { for all } 1 \leqslant i \leqslant \frac{\omega k}{h} .
$$

For each $\hat{B}_{i}$ in $\hat{\mathcal{B}}$, let $\mathcal{B}_{i}$ be a perfect $B$-tiling in $\hat{B}_{i}$ (recall that earlier we chose $s$ and $\lambda$ such that $\hat{B}$ contains a perfect $B$-tiling). Let $\mathcal{B}:=\bigcup \mathcal{B}_{i}$ and observe that $\mathcal{B}$ is a perfect $B$-tiling in $R$. To aid with calculations we will sometimes work with $\mathcal{B}$ instead of $\hat{\mathcal{B}}$.

Let $q^{\prime}:=(1-\varepsilon|\hat{B}|) q$. By Proposition 23 , for all $1 \leqslant i \leqslant \hat{k}$ we can remove $\varepsilon|\hat{B}| q$ vertices from each cluster $V_{a}$ belonging to $\hat{B}_{i}$ so that each edge $V_{a} V_{b}$ in $\hat{B}_{i}$ now corresponds to a $(2 \varepsilon, d / 2)$-superregular pair $\left(V_{a}, V_{b}\right)_{G^{\prime}}$. Further, all clusters now have size $q^{\prime}$ and for each edge $V_{a} V_{b}$ in $\hat{B}_{i}$ the pair $\left(V_{a}, V_{b}\right)_{G^{\prime}}$ is a $2 \varepsilon$-regular pair with density at least $d / 2$ (by Fact 20).

Add all the vertices we removed from the clusters to $V_{0}$ and observe that now, since $\varepsilon \ll \eta_{1}, 1 /|\hat{B}|$,

$$
\left|V_{0}\right| \leqslant 3 \eta_{1} n
$$

From now on, we will refer to the subclusters of size $q^{\prime}$ as the clusters of $R$.

By considering a random partition of each cluster $V_{a}$, and applying a Chernoff bound, one can obtain the following partition of each cluster.

Claim 35. Let $V_{a}$ be a cluster. Then there exists a partition of $V_{a}$ into a red part $V_{a}^{\text {red }}$ and a blue part $V_{a}^{\text {blue }}$ such that

$$
|| V_{a}^{\text {red }}|-| V_{a}^{\text {blue }}|| \leqslant \varepsilon q^{\prime}
$$

and

$$
|| N_{G}(x) \cap V_{a}^{\text {red }}|-| N_{G}(x) \cap V_{a}^{\text {blue }}||<\varepsilon q^{\prime} \text { for all } x \in V(G) .
$$


Apply Claim 35 to every cluster to yield a partition of $V(G)-V_{0}$ into red and blue vertices. In the next section, we will remove vertices of particular copies of $H$ in $G$ from their respective clusters and do so in such a way that we avoid all the red vertices of $G$. After removing these vertices, we will be able to conclude that each (modified) pair $\left(V_{a}, V_{b}\right)_{G^{\prime}}$ is $(5 \varepsilon, d / 5)$-superregular ${ }^{4}$ since $V_{a}^{r e d}$ and $V_{b}^{r e d}$ had no vertices removed from them. After the next section, we will only remove a bounded number of vertices from the clusters, which will not affect the superregularity of pairs of clusters in any significant way.

\subsection{Covering the exceptional vertices}

As in [14], given $x \in V_{0}$, we call a copy of $B \in \mathcal{B}$ useful for $x$ if there exist $r-1$ clusters in $B$, each belonging to a different vertex class of $B$, such that $x$ has at least $\alpha q^{\prime}$ neighbours in each cluster. Denote by $k_{x}$ the number of copies of $B$ in $\mathcal{B}$ that are useful for $x$. The following calculation demonstrates that

$$
k_{x} \beta q^{\prime} \geqslant\left|V_{0}\right|
$$

By (2) and (6), we have that

$$
\begin{aligned}
& k_{x}|B| q^{\prime}+\left(|\mathcal{B}|-k_{x}\right)\left(|B| q^{\prime}-(1-\alpha) q^{\prime} \hat{c}(\omega+\sigma)\right) \\
& \geqslant d_{G}(x)-\left|V_{0}\right| \\
& \geqslant\left(1-\frac{\omega+\sigma}{h}+\frac{\eta}{2}\right) q^{\prime}|B||\mathcal{B}|
\end{aligned}
$$

which implies

$$
\left(|\mathcal{B}|-k_{x}\right)\left(-(1-\alpha) q^{\prime} \hat{c}(\omega+\sigma)\right) \geqslant\left(-\frac{\omega+\sigma}{h}+\frac{\eta}{2}\right) q^{\prime} h \hat{c}|\mathcal{B}|
$$

Rearranging, we get

$$
k_{x} \geqslant \frac{|\mathcal{B}|\left(\frac{h \eta}{2}-\alpha(\omega+\sigma)\right)}{(\omega+\sigma)(1-\alpha)} .
$$

Since $\alpha \ll \eta$, we have that

$$
k_{x} \geqslant \frac{\eta|\mathcal{B}|}{4} .
$$

Now as $|\mathcal{B}| q^{\prime} \geqslant \frac{n}{2|B|}$ and $\eta_{1} \ll \beta, \eta, 1 / h$ we have that

$$
k_{x} \beta q^{\prime} \geqslant \eta|\mathcal{B}| \beta q^{\prime} / 4>3 \eta_{1} n \geqslant\left|V_{0}\right| .
$$

Hence we can assign greedily each vertex $x \in V_{0}$ to a copy $B_{x}$ that is useful for $x$ and do so in such a way that at most $\beta q^{\prime}$ vertices in $V_{0}$ are assigned to the same copy $B \in \mathcal{B}$.

${ }^{4}$ Where $V_{a} V_{b}$ is any edge in any $\hat{B}_{i}$ in $\hat{\mathcal{B}}$. 
Then for each copy $B_{x} \in \mathcal{B}$ that is useful for some $x \in V_{0}$ we can apply Lemma 21 to find a copy of $H$ containing $x$ which contains no red vertices. We do this as follows:

For each $x$, since $\varepsilon \ll \alpha$ and $x$ has at least $\alpha q^{\prime}$ neighbours in $r-1$ clusters belonging to different vertex classes of $B_{x}$, Claim 35 implies that $x$ has at least $\alpha q^{\prime} / 4$ blue neighbours in each of these $r-1$ clusters. Further, we can find $\alpha q^{\prime} / 4$ blue vertices in a cluster belonging to the vertex class of $B_{x}$ that does not necessarily contain any neighbours of $x$. Then it is easy to see that we can find subclusters $S_{1}, \ldots, S_{r}$ of $r$ clusters in $B_{x}$ such that: all vertices in $S_{1} \cup \ldots \cup S_{r}$ are blue vertices; $\left|S_{i}\right|=\alpha q^{\prime} / 4$ for each $i$; every vertex in $S_{1} \cup \ldots \cup S_{r-1}$ is a neighbour of $x$ in $G$. By Fact 20 , each pair $\left(S_{i}, S_{j}\right), 1 \leqslant i<j \leqslant r$, corresponds to an $(8 \varepsilon / \alpha)$-regular

pair in $G^{\prime}$ with density at least $d / 3$. Using Lemma 21 with parameters $8 \varepsilon / \alpha, d / 3, \alpha q^{\prime} / 4$, $h-1$, we find a copy of $H$ containing $x$. Since each $B \in \mathcal{B}$ has been assigned to at most $\beta q^{\prime}$ vertices in $V_{0}$ and $\beta \ll \alpha$ (from (2)), we may repeat the above argument to find copies of $H$ that contain each exceptional vertex in such a way that the copies are disjoint and contain no red vertices. Denote the $H$-tiling induced by these copies of $H$ by $\mathcal{H}_{1}$. Remove all the vertices lying in these copies of $H$ from their respective clusters. Observe that currently, for each $i$,

$$
(1-\beta h) q^{\prime} \leqslant\left|V_{i}\right| \leqslant q^{\prime}
$$

\subsection{Making the blow-up of each $B \in \mathcal{B}$ divisible by $h$}

For a subgraph $S \subseteq R$, let $V_{G}(S)$ denote the union of the clusters in $S$. We aim to apply Lemma 24 to each $\hat{B}_{i}$ in $\hat{\mathcal{B}}$ to find an $H$-tiling that covers every vertex of $V_{G}\left(\hat{B}_{i}\right)$. Combining these $H$-tilings with $\mathcal{H}_{1}$ will result in a perfect $H$-tiling in $G$ as desired. Recall that, for each $1 \leqslant i \leqslant \hat{k}, \hat{G}_{i}$ is the $r$-partite subgraph of $G^{\prime}$ whose $j$ th vertex class is the union of all those clusters contained in the $j$ th vertex class of $\hat{B}_{i}$, for each $1 \leqslant j \leqslant r$. Further, recall that $G_{i}^{*}$ is the complete $r$-partite graph on the same vertex set as $\hat{G}_{i}$. To apply Lemma 24 to each $\hat{B}_{i}$ in $\hat{\mathcal{B}}$ we require that each $G_{i}^{*}$ contains a perfect $H$-tiling. To guarantee the existence of these perfect $H$-tilings we will apply Lemma 26 . To use Lemma 26 on $G_{i}^{*}$ we require that $\left|V\left(\hat{G}_{i}^{*}\right)\right|$ is divisible by $h$. When we first chose our $\hat{B}$ tiling this was the case. Indeed, as each $\hat{B}_{i}$ contained a perfect $H$-tiling and every cluster $V_{i}$ was the same size, $\left|V\left(G_{i}^{*}\right)\right|$ was divisible by $h$. However, in the last section we took out vertices from $G$ in a greedy way, changing the sizes of the clusters in $R$. Hence we cannot guarantee that $\left|V\left(G_{i}^{*}\right)\right|$ is still divisible by $h$ for each $i$. Now we will take out a further bounded number of copies of $H$ in $G$ to ensure $\left|V\left(G_{i}^{*}\right)\right|$ is divisible by $h$ for each $1 \leqslant i \leqslant \hat{k}$. In fact, we will ensure $\left|V_{G}(B)\right|$ is divisible by $h$ for each $B \in \mathcal{B}$.

We now split into two cases: when $r \geqslant 3$ and when $r=2$. When $r \geqslant 3$ we have that $\operatorname{hcf}_{\chi}(H)=1$ and this property will be central to our argument. For $r=2$, we have that $\operatorname{hcf}_{c}(H)=1$ and $\operatorname{hcf}_{\chi}(H) \leqslant 2$. The former property will provide us an easy way of removing copies of $H$ from $V(G)$ to ensure $\left|V_{G}(B)\right|$ is divisible by $h$ for each $B \in \mathcal{B}$. Further, we will not need to use the property that $\operatorname{hcf}_{\chi}(H) \leqslant 2$ in our argument. The only 
time we (implicitly) use the property that $\operatorname{hcf}_{\chi}(H) \leqslant 2$ will be when we apply Lemma 26 .

\subsubsection{Case 1: $r \geqslant 3$}

For a subgraph $S$ of $R$, let $V_{R}(S)$ denote the vertex set of $S$. To assist in our argument, we define an auxiliary graph $F$ whose vertices are the copies of $B$ in $\mathcal{B}$ and for $B_{1}, B_{2} \in V(F)$, we let $B_{1} B_{2}$ be an edge in $F$ if and only if there exists a vertex $x$ in $V_{R}\left(B_{1}\right)$ and $r-1$ vertices in $V_{R}\left(B_{2}\right)$, all in different vertex classes of $B_{2}$, (or vice versa) such that these $r$ vertices induce a $K_{r}$ in $R$. Assume $F$ is connected and let $B_{1} B_{2}$ be an edge in $F$. Then we may apply Lemma 21 to find $h-1$ disjoint copies of $H$ which each have one vertex in $V_{G}\left(B_{1}\right)$ and all other vertices in $V_{G}\left(B_{2}\right)$ (or vice versa). This means that we can remove at most $h-1$ copies of $H$ to ensure $V_{G}\left(B_{1}\right)$ is divisble by $h$. Continuing in this way we can 'shift the remainders mod $h$ ' along a spanning tree of $F$ to ensure $\left|V_{G}(B)\right|$ is divisible by $h$ for each $B \in \mathcal{B}$. (Indeed, since $n$ is divisible by $h$ we have that $\sum_{B \in \mathcal{B}}\left|V_{G}(B)\right|$ is divisible by $h$.)

So assume $F$ is not connected. Let $\mathcal{C}$ be the set of all components of $F$. For $C \in \mathcal{C}$ we will write $V_{R}(C)$ for the set of vertices in $R$ belonging to copies of $B$ in $C$ and $V_{G}(C)$ for the union of the clusters corresponding to the vertices in $V_{R}(C)$. In what follows our aim is to remove a bounded number of copies of $H$ to ensure that for each component $C \in \mathcal{C}$ we have that $\left|V_{G}(C)\right|$ is divisible by $h$. Then we can apply our previous argument to spanning trees of each component to achieve that $\left|V_{G}(B)\right|$ is divisible by $h$ for each $B \in \mathcal{B}$.

Call vertices in $R$ of degree at least

$$
(1-\omega / h+\eta / 4) k
$$

big. If a vertex is not big, call it small. Note by (5) that all but at most $\omega k / h-1$ vertices in $R$ are big.

Claim 36. Let $C_{1}, C_{2} \in \mathcal{C}, C_{1} \neq C_{2}$ and let $a \in V_{R}\left(C_{2}\right)$. Then

$$
\left|N_{R}(a) \cap V_{R}\left(C_{1}\right)\right|<\left(1-\frac{\omega+\sigma}{h}+\frac{\eta}{4}\right)\left|V_{R}\left(C_{1}\right)\right| .
$$

Proof. Recall that $B$ has width $\omega \hat{c}$. Suppose Claim 36 is false. Then there exists some $B_{0} \in \mathcal{B}$ such that $B_{0} \in C_{1}$ and

$$
\left|N_{R}(a) \cap B_{0}\right| \geqslant\left(1-\frac{\omega+\sigma}{h}+\frac{\eta}{4}\right)\left|B_{0}\right|=(r-2) \omega \hat{c}+\frac{\eta h \hat{c}}{4} .
$$

Thus $a$ must have neighbours in at least $r-1$ vertex classes of $B_{0}$. We can therefore construct a copy of $K_{r}$ in $R$ which consists of $a$ together with $r-1$ of its neighbours in $B_{0}$. But by definition of the auxiliary graph $F$, we must have that $B_{0}$ is adjacent in $F$ to the copy of $B$ in $\mathcal{B}$ that contains $a$. This contradicts that $C_{1}$ and $C_{2}$ were different components of $F$. Thus Claim 36 holds. 
Claim 37. There exist components $C_{1}, C_{2} \in \mathcal{C}, C_{1} \neq C_{2}$, a big vertex $x_{1} \in V(R)$ and another (not necessarily big) vertex $x_{2} \in V(R)$ such that $x_{1} \in V\left(C_{1}\right), x_{2} \in V\left(C_{2}\right)$ and $x_{1} x_{2} \in E(R)$.

Proof. Take some big vertex $x \in V(R)$. Then $x$ is in $V_{R}\left(C_{x}\right)$ for some component $C_{x}$ of $F$. We have $\left|C_{x}\right| \geqslant(1-\omega / h+\eta / 4) k$, as otherwise $x$ has a neighbour in $R$ outside of $C_{x}$ and we are done. Since $r \geqslant 3$,

$$
\left|R \backslash V_{R}\left(C_{x}\right)\right| \leqslant(\omega / h-\eta / 4) k<(1-\omega / h+\eta / 4) k .
$$

If $R \backslash V_{R}\left(C_{x}\right)$ contains any big vertex $y$, then $y$ has a neighbour in $V_{R}\left(C_{x}\right)$ since $\left|R \backslash V_{R}\left(C_{x}\right)\right|<(1-\omega / h+\eta / 4) k$ and we are done. Hence assume all big vertices are in $V_{R}\left(C_{x}\right)$. Then all vertices in $R \backslash V_{R}\left(C_{x}\right)$ are small vertices. Let $z$ be a small vertex in $R \backslash V_{R}\left(C_{x}\right)$. Since $r \geqslant 3$,

$$
d_{R}(z) \geqslant(1-(\omega+\sigma) / h+\eta / 4) k \geqslant(\omega / h+\eta / 4) k .
$$

Since there are at most $\omega k / h-1$ small vertices in $R$, we have that $z$ has a neighbour $w$ which is a big vertex. But then $w \in V_{R}\left(C_{x}\right)$. Thus Claim 37 holds.

Claim 38. There exists a copy $K^{\prime}$ of $K_{r}$ in $R$ which has vertices in at least two components of $F$.

Proof. By Claim 37, there exist components $C_{1}, C_{2} \in \mathcal{C}$, a big vertex $x_{1} \in V(R)$ and another vertex $x_{2} \in V(R)$ such that $x_{1} \in V_{R}\left(C_{1}\right), x_{2} \in V_{R}\left(C_{2}\right)$ and $x_{1} x_{2} \in E(R)$. By (5) and (7), $x_{1}$ and $x_{2}$ have a common neighbourhood of size at least

$$
((r-3) \omega / h+\eta / 2) k
$$

If $r=3$, then we choose $x_{3}$ in the common neighbourhood of $x_{1}$ and $x_{2}$, and we are done. So assume $r \geqslant 4$. Since there are at most $\omega k / h$ small vertices, we can choose a big vertex $x_{3}$ in the common neighbourhood of $x_{1}$ and $x_{2}$. Then $x_{1}, x_{2}$ and $x_{3}$ have a common neighbourhood of size at least

$$
((r-4) \omega / h+3 \eta / 4) k
$$

If $r=4$, then we choose $x_{4}$ in the common neighbourhood of $x_{1}, x_{2}$ and $x_{3}$ and we are done. Otherwise $r \geqslant 5$ and we continue as before. Thus Claim 38 holds.

For such a copy $K^{\prime}$ of $K_{r}$ in $R$, we now show that we can take out a bounded number of copies of $H$ from the clusters corresponding to the vertices of $K^{\prime}$ in such a way that that leaves one of the components $C \in \mathcal{C}$ with $\left|V_{G}(C)\right|$ divisible by $h$. We use Theorem 30 and Lemma 21 to achieve this. We will then repeat this process to ensure $\left|V_{G}(B)\right|$ is divisible by $h$ for each $B \in \mathcal{B}$.

Claim 39. There exists $t \in \mathbb{N}$ such that by removing at most $t+(|\mathcal{B}|-|\mathcal{C}|)(h-1)$ copies of $H$ from $G$ we can ensure $\left|V_{G}(B)\right|$ is divisible by $h$ for each $B \in \mathcal{B}$. 
Proof. Firstly, for each component $C \in \mathcal{C}$ we will remove copies of $H$ to ensure $\left|V_{G}(C)\right|$ is divisible by $h$. Apply Claim 38 to find a copy $K^{\prime}$ of $K_{r}$ in $R$ which has vertices in at least two components of $F$. Let $C^{*}$ be a component of $F$ which contains at least one vertex of $K^{\prime}$. Let $p$ be the number of vertices of $K^{\prime}$ contained in $C^{*}$ and observe that $1 \leqslant p \leqslant r-1$. Let $0 \leqslant g \leqslant h-1$ such that $\left|V_{G}\left(C^{*}\right)\right| \equiv g \bmod h$. If $g=0$ then $\left|V_{G}\left(C^{*}\right)\right|$ is divisible by $h$ and we consider the graphs $F_{1}:=F-V\left(C^{*}\right)$ and $R_{1}:=R-V_{R}\left(C^{*}\right)$. So assume $1 \leqslant g \leqslant h-1$. Observe that we can apply Lemma 21 to find any bounded number of disjoint copies of $H$ in $G$ in the clusters of $K^{\prime}$ (see the end of Section 7.2). For any copy $H^{\prime}$ of $H$ in $G$ in the clusters of $K^{\prime}$ there are precisely $p$ colour classes of some colouring $c$ of $H^{\prime}$ contained in the clusters of $K^{\prime}$ in $V_{G}\left(C^{*}\right)$. Moreover, given any colouring $c$ of $H$ and $p$-subset $P$ contained in $D_{c}$ (recall Definition 29) we can find any bounded number of disjoint copies $H^{\prime}$ of $H$ in $G$ with colouring $c$ in the clusters of $K^{\prime}$ so that the colour classes of $H^{\prime}$ in $V_{G}\left(C^{*}\right)$ correspond to the $p$-subset $P$. So there exists $j \in\left\{1, \ldots, z_{p}\right\}$ such that $P=A_{p, c, j}$ (recall this notation from Definition 29). Thus, removing such a copy $H^{\prime}$ of $H$ from $G$ would result in removing precisely $S_{p, c, j}$ vertices from $V_{G}\left(C^{*}\right)$. By Theorem 30, there exist a collection of non-negative integers $\left\{a_{p, c, i}: c \in C_{H}, 1 \leqslant i \leqslant z_{p}\right\}$ and $\bar{a} \in \mathbb{N}$ such that

$$
a_{p, c, i} \leqslant \bar{a} \text { for all } c \in C_{H}, 1 \leqslant i \leqslant z_{p},
$$

and

$$
g \cdot \sum_{c \in C_{H}} \sum_{i=1}^{z_{p}} a_{p, c, i} S_{p, c, i} \equiv g \quad \bmod h .
$$

Hence we can remove

$$
g \cdot \sum_{c \in C_{H}} \sum_{i=1}^{z_{p}} a_{p, c, i} \leqslant(h-1) \bar{a}\left|C_{H}\right| z_{p}
$$

suitable disjoint copies of $H$ in $G$ in the clusters of $K^{\prime}$ to make $\left|V_{G}\left(C^{*}\right)\right|$ divisible by $h$.

Next we consider graphs $F_{1}:=F-V\left(C^{*}\right)$ and $R_{1}:=R-V_{R}\left(C^{*}\right)$. Let $k_{1}:=\left|R_{1}\right|$. Claim 36 and (5) together give us that $R_{1}$ has degree sequence $d_{R_{1}, 1} \leqslant \ldots \leqslant d_{R_{1}, k_{1}}$ where

$$
d_{R_{1}, i} \geqslant\left(1-\frac{\omega+\sigma}{h}\right) k_{1}+\frac{\sigma}{\omega} i+\frac{\eta k_{1}}{4} \text { for all } 1 \leqslant i \leqslant \frac{\omega k_{1}}{h} .
$$

Suppose $|\mathcal{C}| \geqslant 3$. Arguing as in Claims 37 and 38 we can find a copy $K_{1}^{\prime}$ of $K_{r}$ in $R_{1}$ which has vertices in at least two components of $F_{1}$.

Let $C^{* *}$ be a component of $F$ which contains at least one vertex of $K_{1}^{\prime}$. As before by removing at most $(h-1) \bar{a}\left|C_{H}\right| z_{p}$ copies of $H$ from the clusters of $K_{1}^{\prime}$ we can make $\left|V_{G}\left(C^{*}\right)\right|$ divisible by $h$. Since $|G|$ is divisible by $h$, we can continue in this way to make $\left|V_{G}(C)\right|$ divisible by $h$ for each component $C \in \mathcal{C}$. We then apply the 'shifting the remainders mod $h$ ' argument mentioned earlier during the ' $F$ connected' case to guarantee that $|B|$ is divisible by $h$ for each $B \in \mathcal{B}$. In this process we removed at most $(|\mathcal{C}|-1)(h-1) \bar{a}\left|C_{H}\right| z_{p}$ disjoint copies of $H$ from $G$. Each time we use the 'shifting the remainders mod $h$ ' argument on a connected component $C \in \mathcal{C}$ we remove at most $(|C|-1)(h-1)$ disjoint copies of $H$ in $G$. Hence overall we remove at most $(|\mathcal{C}|-1)(h-1) \bar{a}\left|C_{H}\right| z_{p}+(|\mathcal{B}|-|\mathcal{C}|)(h-1)$ disjoint copies of $H$ in $G$. Denote this $H$-tiling (formed from these copies of $H$ ) by $\mathcal{H}_{2}$. 
Observe that now

$$
(1-2 h \beta) q^{\prime} \leqslant\left|V_{i}\right| \leqslant q^{\prime}
$$

for each $i$ since we only removed a bounded number of vertices from $G$.

\subsubsection{Case 2: $r=2$}

As in the statement of Theorem 30, let $b$ be the number of components of $H$ and $t_{1}, \ldots, t_{b}$ be the sizes of the components of $H$. By Theorem 30, there exists a collection of nonnegative integers $\left\{a_{i}: 1 \leqslant i \leqslant b\right\}$ and $\bar{a} \in \mathbb{N}$ such that

$$
a_{i} \leqslant \bar{a} \text { for all } 1 \leqslant i \leqslant b,
$$

and

$$
\sum_{i=1}^{b} a_{i} t_{i} \equiv 1 \quad \bmod h .
$$

Let $B_{1}, B_{2} \in \mathcal{B}$. If $\left|V_{G}\left(B_{1}\right)\right| \equiv 0 \bmod h$, define $\mathcal{B}_{1}:=\mathcal{B} \backslash B_{1}$. If not, let $p \in\{1, \ldots, h-1\}$ such that $\left|V_{G}\left(B_{1}\right)\right| \equiv p \bmod h$. Remove $p \sum_{i=1}^{b} a_{i}$ copies of $H$ from $V_{G}\left(B_{1}\right) \cup V_{G}\left(B_{2}\right)$ in the following way: For each $1 \leqslant i \leqslant b$, remove $p a_{i}$ copies of $H$ from $V_{G}\left(B_{1}\right) \cup V_{G}\left(B_{2}\right)$ such that the component of order $t_{i}$ is in $V_{G}\left(B_{1}\right)$ and all other components are in $V_{G}\left(B_{2}\right){ }^{5}$ Since $p \sum_{i=1}^{b} a_{i} t_{i} \equiv p \bmod h$, by removing these $p \sum_{i=1}^{b} a_{i}$ copies of $H$ from $V_{G}\left(B_{1}\right) \cup V_{G}\left(B_{2}\right)$ we now have that $\left|V_{G}\left(B_{1}\right)\right|$ is divisible by $h$. Define $\mathcal{B}_{1}:=\mathcal{B} \backslash B_{1}$.

Let $B_{1}^{\prime}, B_{2}^{\prime} \in \mathcal{B}_{1}$. If $\left|V_{G}\left(B_{1}^{\prime}\right)\right| \equiv 0 \bmod h$, define $\mathcal{B}_{2}:=\mathcal{B}_{1} \backslash B_{1}^{\prime}$. If not, let $p^{\prime} \in$ $\{1, \ldots, h-1\}$ such that $\left|V_{G}\left(B_{1}^{\prime}\right)\right| \equiv p^{\prime} \bmod h$. Remove $p^{\prime} \sum_{i=1}^{b} a_{i}$ copies of $H$ from $V_{G}\left(B_{1}^{\prime}\right) \cup V_{G}\left(B_{2}^{\prime}\right)$ in the same way as before. Define $\mathcal{B}_{2}:=\mathcal{B}_{1} \backslash B_{1}^{\prime}$. Continuing in the same way, we see that by removing at most

$$
(|\mathcal{B}|-1)(h-1) b \bar{a}
$$

copies of $H$ we can ensure that $|B|$ is divisible by $h$ for each $B \in \mathcal{B}$. Denote this $H$-tiling (formed from these copies of $H$ ) by $\mathcal{H}_{2}$.

Observe that now

$$
(1-2 h \beta) q^{\prime} \leqslant\left|V_{i}\right| \leqslant q^{\prime}
$$

for each $i$ since we only removed a bounded number of vertices.

\subsection{Completing the perfect tiling}

As we related at the beginning of Section 7.3, we aim to apply Lemma 24 to each $\hat{B}_{i} \subseteq R$ $(1 \leqslant i \leqslant \hat{k})$ where the vertices of $R$ are the now modified clusters - modified by the removing of copies of $H$ in previous sections. Recall that, for each $1 \leqslant i \leqslant \hat{k}, \hat{G}_{i}$ is the $r$-partite subgraph of $G^{\prime}$ whose $j$ th vertex class is the union of all those clusters contained

\footnotetext{
${ }^{5}$ We use Lemma 21 to do this.
} 
in the $j$ th vertex class of $\hat{B}_{i}$, for each $1 \leqslant j \leqslant r$. Observe that in Section 7.3 we made $\left|\hat{G}_{i}\right|=\left|V_{G}\left(\hat{B}_{i}\right)\right|$ divisible by $h$ for each $i$. Further,

$$
(1-2 h \beta) q^{\prime} \leqslant\left|V_{i}\right| \leqslant q^{\prime}
$$

for each $i$. Recall that $G_{i}^{*}$ is the complete $r$-partite graph on the same vertex set as $\hat{G}_{i}$. Since $0<2 h \beta \ll \sigma / \omega, 1-\sigma / \omega, 1 / h$ by $(2)$, we can apply Lemma 26 to conclude that each $G_{i}^{*}$ contains a perfect $H$-tiling.

Furthermore, pairs of clusters that correspond to edges of $\hat{B}_{i}$ are still $(6 \varepsilon, d / 6)$ superregular. Indeed, in Section 7.2 we removed copies of $H$ which avoided red vertices, resulting in each pair of clusters (in a copy of $H$ ) being $(5 \varepsilon, d / 5)$-superregular. Then, in Section 7.3.1, or Section 7.3.2 if $r=2$, we removed only a constant number of vertices from each cluster. Hence each pair of clusters (in a copy of $H$ ) is $(6 \varepsilon, d / 6)$-superregular.

We now have all we need to apply Lemma 24 to find a perfect $H$-tiling $\hat{\mathcal{H}}_{i}$ in $\hat{G}_{i}$ for each $1 \leqslant i \leqslant \hat{k}$. Then

$$
\mathcal{H}_{1} \cup \mathcal{H}_{2} \cup \hat{\mathcal{H}}_{1} \cup \ldots \cup \hat{\mathcal{H}}_{\hat{k}}
$$

is a perfect $H$-tiling in $G$. Hence we have proved Theorem 9 .

\section{Acknowledgements}

The first author would like to thank Pat Devlin for a helpful conversation at Building Bridges II. The authors are also grateful to the referees for their helpful and careful reviews.

\section{References}

[1] N. Alon and R. Yuster, H-factors in dense graphs, J. Combin. Theory Ser. B 66 (1996), 269-282.

[2] J. Balogh, A.V. Kostochka and A. Treglown, On perfect packings in dense graphs, Electron. J. Combin. 20(1) (2013), \#P57.

[3] D. Bauer, H.J. Broersma, J. van den Heuvel, N. Kahl, A. Nevo, E. Schmeichel, D.R. Woodall and M. Yatauro, Best Monotone Degree Conditions for Graph Properties: A Survey, Graphs Combin. 31 (2015), 1-22.

[4] V. Chvátal, On Hamilton's ideals, J. Combin. Theory Ser. B 12 (1972), 163-168.

[5] A. Hajnal and E. Szemerédi, Proof of a conjecture of Erdős, Combinatorial Theory and its Applications vol. II 4 (1970), 601-623.

[6] P. Hell and D.G. Kirkpatrick, On the complexity of general graph factor problems, SIAM J. Computing 12 (1983), 601-609.

[7] J. Hyde, H. Liu and A. Treglown, A degree sequence Komlós theorem, SIAM J. Discrete Math. 33 (2019), 2041-2061.

[8] S. Janson, T. Luczak and A. Ruciński, Random graphs, John Wiley ES Sons, 2010. 
[9] H.A. Kierstead and A.V. Kostochka, An Ore-type Theorem on Equitable Coloring, J. Combin. Theory Ser. B 98 (2008), 226-234.

[10] J. Komlós, Tiling Turán Theorems, Combinatorica 20 (2000), 203-218.

[11] J. Komlós, G.N. Sárközy and E. Szemerédi, Blow-up lemma, Combinatorica 17(1) (1997), 109-123.

[12] J. Komlós, G.N. Sárközy and E. Szemerédi, Proof of the Alon-Yuster conjecture, Discrete Math. 235 (2001), 255-269.

[13] J. Komlós and M. Simonovits, Szemerédi's Regularity Lemma and its applications in graph theory, Combinatorics: Paul Erdös is eighty vol. II (1996), 295-352.

[14] D. Kühn and D. Osthus, Critical chromatic number and the complexity of perfect packings in graphs, 17th ACM-SIAM Symposium on Discrete Algorithms (SODA 2006), 851-859.

[15] D. Kühn and D. Osthus, The minimum degree threshold for perfect graph packings, Combinatorica 29 (2009), 65-107.

[16] D. Kühn, D. Osthus and A. Taraz, Large planar subgraphs in dense graphs, J. Combin. Theory Ser. B 95(2) (2005), 263-282.

[17] C.St.J.A. Nash-Williams, Hamiltonian arcs and circuits, in Recent Trends in Graph Theory. Lecture Notes in Mathematics, Springer, Berlin 186 (1971), 197-210.

[18] A. Shoukoufandeh and Y. Zhao, Proof of a conjecture of Komlós, Random Struct. Alg. 23 (2003), 180-205.

[19] E. Szemerédi, Regular partitions of graphs, Problémes Combinatoires et Théorie des Graphes Colloques Internationaux CNRS 260 (1978), 399-401.

[20] A. Treglown, A degree sequence Hajnal-Szemerédi theorem, J. Combin. Theory Ser. B 118 (2016), 13-43. 